\title{
An extended Marciniak-Kuczynski model for anisotropic sheet subjected to monotonic strain paths with through-thickness shear
}

\author{
Philip Eyckens ${ }^{*, a}$, Albert Van Bael ${ }^{\mathrm{a}, \mathrm{b}}$ and Paul Van Houtte ${ }^{\mathrm{a}}$ \\ a Department MTM, Katholieke Universiteit Leuven, Kasteelpark Arenberg 44 Bus 02450, \\ BE-3001 Heverlee, Belgium \\ ${ }^{\mathrm{b}}$ IWT, KHLim (Limburg Catholic University College), Campus Diepenbeek, Agoralaan \\ Gebouw B, Bus 3, BE-3590 Diepenbeek, Belgium
}

\begin{abstract}
Some metal sheet forming processes may induce an amount of plastic shear over the sheet thickness. This paper investigates how formability of anisotropic sheet metal is affected by such Through-Thickness Shear (TTS). The Marciniak-Kuczynski (MK) model framework, a commonly used analytical tool to predict the limit of sheet formability due to the onset of localized necking, is extended in this paper in order to explicitly account for TTS in anisotropic metal sheets. It is a continuation of previous work by the present authors ((Eyckens et al., 2009)), in which TTS is incorporated for isotropic sheet. This is achieved by the introduction of additional force equilibrium and geometric compatibility equations that govern the connection between matrix and groove in the MK model. Furthermore, in order to integrate plastic anisotropy, a material reference frame available in recent literature is incorporated, as well as a particular model for anisotropic yielding that relies on virtual testing of anisotropic properties (Facet plastic potential), since out-of-plane anisotropy related to TTS cannot be measured experimentally.
\end{abstract}


It is found that formability may be increased by TTS, depending on the direction onto which it is imposed by the forming process. TTS is thus a relevant aspect of the formability in, for instance, sheet forming processes in which sliding contact with friction between sheet and forming tools occur.

Keywords: fracture mechanisms (A), metallic material (B), plates (B), stability and bifurcation (C), Forming Limit Curve, texture-induced anisotropy

* Corresponding author. Philip.eyckens@mtm.kuleuven.be; Tel.: +32 16321305; fax: +32 16321990. 


\section{Introduction}

\subsection{State-of-the-art}

Localized necking is the most studied mechanism limiting the formability during sheet metal forming processes. This plastic instability can be modelled by some different theoretic approaches, such as bifurcation theory (Hashiguchi and Protasov, 2004), perturbation theory (Boudeau et al., 1998) and the shear instability criterion proposed in (Bressan and Williams, 1983). The best known and most widely spread approach is known as the MarciniakKuczynski (MK) model (Marciniak and Kuczynski, 1967). The currently developed theory is part of ongoing worldwide research to use the MK model framework to gain insights in fundamental effects of various material as well as process properties on the onset of localized necking in sheet metal forming processes. The following literature review is restricted to the last few years, though research on this topic is in fact much older, see e.g. Ratchev et al. (1994). A more elaborate review on the modelling approaches and developments in the last half century is included in the authors' preceding paper (Eyckens et al., 2008).

State-of-the-art material models are currently employed in the MK framework to improve prediction capability. In (Ahmadi et al., 2009), it is shown that the use of more advanced phenomenological yield functions, i.e. requiring a greater number of experimentally determined yield points, significantly improves the quality of prediction of the Forming Limit Curve (FLC) based on the MK model. Considering distortional hardening (i.e. a change in yield locus shape as a consequence of texture evolution) on a phenomenological basis has also been found to influence MK formability predictions ((Aretz, 2008)). Other research uses plasticity models to describe material behaviour in the MK model, aiming to reduce the number of mechanical tests. For instance, (Charca Ramos et al., In Press) adopts 
the ViscoPlastic Self-Consistent (VPSC) polycrystal model, resulting in a satisfying FLC prediction. In (Signorelli et al., 2009), the capability of the VPSC scheme to consider crystallographic texture evolution, and its resulting texture hardening/softening, is seen to influence the right-hand side of the FLD. A similar conclusion is drawn in (Yoshida et al., 2009) for the plane strain deformation mode, employing the Taylor polycrystal model.

The preceding papers apply to materials with dislocation glide as the plastic deformation mechanism. Materials with other deformation mechanisms have also received attention in conjunction with the MK theory, e.g. (Makkouk et al., 2008) for stainless steel (TRIP TRansformation Induced Plasticity), (Chung et al., In Press) for TWIP (Twinning Induced Plasticity) steels, and (Neil and Agnew, 2009) and (Lévesque et al., 2010) for HCP materials that deform by combined twinning and dislocation glide.

Other recent papers are dedicated to extending the applicability of the MK model framework to process-specific forming conditions. The MK model can be used as a postprocessing tool of finite element simulations of forming processes, explicitly taking into account the large influence of strain-path changes on formability ((Barata da Rocha et al., 2009)). The anisotropic hardening associated with strain-path changes plays an important role on the subsequent formability as shown in e.g. (Yoshida and Suzuki, 2008). In forming operations in which the sheet normal compressive stress is significant, such as in hydro forming, formability is generally larger, which has been demonstrated theoretically by extensions of the MK model framework in (Banabic and Soare, 2008), (Wu et al., 2009) and (Assempour et al., 2010).

Recently, an extension of the MK model has been proposed in (Allwood and Shouler, 2009) that accounts for (i) a non-negligible sheet normal stress, and/or (ii) non-negligible through-thickness shear stress. Since the point (ii) of this MK extension corresponds to the 
extension proposed in this paper, it is discussed in more detail and compared to the current model in section 2.11.

\subsection{Outline of the proposed model}

The present authors have recently proposed an extension to the MK model to incorporate Through-Thickness Shear (TTS) during in-plane deformation for a von Mises material ((Eyckens et al., 2009)). The current paper generalizes this model to anisotropic sheets, through incorporation of a texture-based analytical description of the yield locus called the Facet method ((Van Houtte et al., 2009)).

The theoretical development of this model is given in section 2. At the heart of any MK model lays the 'connection' between two zones ('groove' and 'matrix'), i.e. the geometric compatibility (section 2.5) on the one hand, and force equilibrium (section 2.6) on the other hand. The incorporation of TTS requires some new fundamental equations of both types. They have been conceived on a physical ground, cf. their detailed description in the sections 2.5-2.6. Besides these fundamental changes of the MK model framework, care must also be taken regarding the description of the mechanical behaviour. As it is aimed to predict formability of an anisotropic material under strain paths that contain shear (more in particular through-thickness shear), two additional fundamental questions regarding material behaviour arise that usually are not encountered in an MK analysis:

(i) How to express the anisotropy for materials under shearing?

A body-fixed Cartesian reference frame (i.e. with axes along material fibres) loses its orthogonality under the influence of shearing, thus becoming non-Cartesian. However, yield criteria are typically formulated in a Cartesian reference frame. This is actually a classical problem for large-strain finite element modelling, where it is usually solved by introducing a 
so-called local (or co-rotational) reference frame, which is supposed to follow the rotation of the material while remaining Cartesian. The continuum-mechanical solutions of Jaumann and Green-Naghdi are probably the best known examples of local frame definitions (both are reviewed in (de Montleau et al., 2008)). Another class of local frames dedicated to metal forming, is based on a definition of the Mandel spin, i.e. the average lattice spin of a polycrystal aggregate, as in (Van Houtte, 2001) and (Duchêne et al., 2008).

A second common feature of the present MK model with large-strain FE formulations, is the discrete solution procedure in time of a continuous problem of field equations. This involves assumptions on the deformation during an increment. Also many different time integration schemes have been proposed in the literature, like the Hughes-Winget scheme (Hughes and Winget, 1980) that is implemented in the commercial FE software ABAQUS@ .

The proposed MK model adopts the method that is proposed in (de Montleau et al., 2008) for updated Lagrangian FE formulations. This method actually combines a local frame definition with a time integration scheme. It is called the CSLVG-method by the original authors, which stands for 'Constant Symmetric Local Velocity Gradient'. Indeed, a local frame is defined in such a way that the local velocity gradient, i.e. the one defined from coordinates in the local frame, is constant as well as symmetric during the increment. Because of the symmetry property, the local velocity gradient is identical to the local strain rate tensor, and time integration of the constitutive law takes a very simple form. In (Duchêne et al., 2008), it is seen that the capability of the CSLVG-scheme to predict the rotation of the axes of orthorhombic sample symmetry after a simple shear test, is comparable to schemes adopting the Mandel spin.

The definition of the adopted local frame is elaborated in section 2.10.1. In the present work, this frame also acts as the material reference frame, i.e. the frame in which anisotropy is expressed. 
(ii) How to describe sheet anisotropy in a realistic way?

Many anisotropic yield criteria have been proposed that rely on a series of mechanical tests, the most commonly used being the uniaxial tensile, shear and equibiaxial test. However, none of these tests provides information on the anisotropic properties under the combined loading of in-plane deformation and TTS, so the reliability of the obtained yield locus in this region of the stress space is highly questionable. The currently proposed solution is based on the hierarchical modelling scheme, i.e. through a series of 'virtual tests' using some kind of multilevel model, covering the whole stress space, and onto which an analytical expression with a high number of parameters is fitted. In the present work, one of the simplest multilevel models, i.e. the Full Constraint Taylor model, is used to fit the parameters of a plastic potential in the form of the Facet expression ((Van Houtte et al., 2009)). The fitting procedure is performed separately (before the actual MK analysis). In future, more advanced multilevel models can easily be adopted to increase the accuracy of the representation of anisotropic properties. Although not investigated presently, the Facet method is generic enough to include strain-rate sensitivity and the stress-differential effect. This method is described further in section 2.10.2.

Section 3 presents a number of formability predictions that are obtained from the extended MK model for one aluminium alloy and one steel alloy. Their crystallographic texture (presented in section 3.1) is used in the Taylor-Bishop-Hill multilevel model in order to fit the coefficients of the Facet plastic potential, cf. section 3.2. Then, section 3.3 shows and discusses the effect of the anisotropy of both commercial sheets on forming limit predictions, for strain paths without and with TTS. 


\section{Model description}

In the model description presented hereafter, the Einstein summation convention is generally not adopted, unless stated otherwise. Because of the proposed generalization of the MK model for Through-Thickness Shear (TTS) and anisotropic plastic material behaviour, it is furthermore desirable to introduce tensors and tensor equations where applicable (including strain, strain rate, Cauchy stress, deformation gradient and velocity gradient).

\subsection{Model zones and reference frames}

The generalized Marciniak-Kuczynski model is composed of two zones. The modelled sheet consists of a matrix $a$ that contains an imperfection in the form of a groove $b$, as sketched in

The matrix $a$ represents the defect-free sheet, which is subjected to a homogeneous deformation. The matrix velocity gradient $\mathbf{L}^{a}$ is expressed in a fixed reference frame (called the global reference frame), as elaborated further in section 2.2. This global reference frame is defined to be attached to the sheet throughout the deformation. Its 3 -axis stays aligned with the sheet normal, even if the matrix undergoes Through-Thickness Shear (TTS). Also the 1and 2-axis, lying in the sheet plane, are defined in a particular way. Consider a grid of etched circles on the surface of the matrix. After some amount of deformation, this grid is deformed into ellipses, as shown in

(b). The global 1-direction is now defined to lie along the longer axis of the ellipse. It is called the major in-plane strain direction. The global 2-direction then lies along the shorter axis of the ellipse, and is called the minor in-plane strain direction. Note that these two 
directions do not correspond to principal strain directions in case the matrix is subjected to TTS.

The groove $b$ is the (simplified) model representation of the inhomogeneous nature of real sheets. The inhomogeneity can arise from many different sources, among which can be mentioned the variation of sheet thickness, the spatial distribution of grains and second phase particles (which give rise to an inhomogeneous strength), and inhomogeneities within each grain, such as the spatial distribution of dislocations and solute atoms. Rather than taking this all explicitly into account, a single scalar measure of all types of inhomogeneity is introduced, similar as in the original MK analysis ((Marciniak and Kuczynski, 1967)). This initial imperfection $f_{0}$ is defined as:

$$
f_{0}=\frac{\vartheta_{0}^{b}}{\vartheta_{0}^{a}}
$$

in which $\vartheta_{0}^{x}$ is the initial thickness; the superscript $x$ refers to either the matrix $a$ or groove $b$. The value of $f_{0}$ is the scalar measure of the inhomogeneity of the sheet; a value of $f_{0}=1$ would represent a perfectly homogeneous sheet. In literature, common values of $f_{0}$ are in the range [0.98;0.9999]. The higher its value, the higher are the resulting formability predictions. Its value is either postulated, or determined by an inverse method, i.e. by comparing predictions using different $f_{0}$ to an experimental point on the FLC. Often, the plane strain intercept $\left(\mathrm{FLC}_{0}\right)$ is chosen, as in (Aretz, 2007).

The groove is infinitely long along one direction in the sheet plane, which is called the groove direction $t$. Its perpendicular direction in the sheet plane is denoted the groove normal direction $n$. Along $n$, the groove is conceived to have a finite length. This width of the groove is however not considered in the model. Such particular choice of the shape of the inhomogeneity $b$ is thus similar to the actual appearance of localized necks. 
A second reference frame used in the model is attached to the groove itself (not to the groove material). This groove reference frame $G$ has the n-, t- and 3- directions as its axes. In the undeformed state (

(a)), its orientation with respect to the global reference frame is given by the angle $\Phi_{0}$, going from the 1-axis to the n-axis. For now, the value of $\Phi_{0}$ may be considered to be known; in section 2.9 this issue is discussed further.

As shown in

(b), the groove frame $G$ may rotate around the 3 -axis (sheet normal) as a consequence of the imposed matrix deformation. The groove orientation in a deformed state, determined by the angle $\Phi$, is given by:

$$
\tan (\Phi)=\exp \left(\varepsilon_{11}^{a}-\varepsilon_{22}^{a}\right) \tan \left(\Phi_{0}\right)
$$

The matrix normal strain components along the 1- and 2-direction, denoted $\varepsilon_{11}^{a}$ and $\varepsilon_{22}^{a}$ respectively, are defined in section 2.4 .

Two more reference frames are defined in the proposed model, i.e. the local reference frames of the material within the matrix $a$ (with $1^{A}-, 2^{A}$ - and $3^{A}$-axes), and within the groove $b$ (with $1^{B}-, 2^{B}$ - and $3^{B}$-axes). In the undeformed state (cf.

(a)), they coincide and are aligned along the usual sample reference frame of rolled sheet: the $1^{x}$-axis corresponds to the rolling direction $R D$ of the sheet, the $2^{x}$-axis to the transverse TD direction, and the $3^{x}$-axis to the sheet normal direction $N D(x=a$ or $b)$. In the undeformed state, the alignment of both local reference frames with respect to the global reference frame is given by the angle $\alpha_{R D}$, defined from the major in-plane strain direction (1-axis) towards the sheet rolling direction $\left(1^{X}\right.$-axis). As deformation proceeds, these local reference frames may rotate due to the respective velocity gradients of the matrix $\left(\mathbf{L}^{a}\right)$ or the 


\subsection{Description of the rate of deformation in the matrix}

In its most general form, a velocity gradient $\mathbf{L}$ has 9 independent components. However, the expression of the matrix velocity in the global reference frame $\left[\mathbf{L}^{a}\right]$ takes a more simplified form:

$$
\left[\mathbf{L}^{a}\right]=\left[\begin{array}{ccc}
L_{11}^{a} & 0 & L_{13}^{a} \\
0 & L_{22}^{a} & L_{23}^{a} \\
0 & 0 & L_{33}^{a}
\end{array}\right]
$$

The fact that the components $L_{31}^{a}$ and $L_{32}^{a}$ equal 0 , follows directly from the choice of the global 3-axis to stay normal to the sheet plane throughout the deformation. Writing the definition of these components, it can be seen that:

$$
L_{3 i}^{a} \equiv \frac{\partial v_{3}^{a}}{\partial x_{i}}=0 \quad(i=1,2)
$$

in which $\mathbf{v}^{a}$ is the velocity field of the matrix.

The fact that the components $L_{12}^{a}$ and $L_{21}^{a}$ equal 0 , follows directly from the choice of the global 1- and 2-axis within the sheet plane (cf. the deformation of the circular grid in ). Expressed in the global reference frame, the matrix thus does not undergo any in-plane shear strain nor in-plane rigid body rotation, which means that: 


$$
L_{12}^{a}=L_{21}^{a}=0
$$

The symmetric and anti-symmetric parts of $\left[\mathbf{L}^{a}\right]$ give the strain rate tensor of the matrix $\left[\mathbf{D}^{a}\right]$ and the tensor of rigid body rotation rate of the matrix $\left[\mathbf{W}^{a}\right]$, respectively. They are both expressed in the global reference frame. From equation (3), they can be written in the following way:

$$
\begin{gathered}
{\left[\mathbf{D}^{a}\right]=\left[\begin{array}{ccc}
1 & 0 & \rho_{13} \\
0 & \rho_{22} & \rho_{23} \\
\rho_{13} & \rho_{23} & -\left(1+\rho_{22}\right)
\end{array}\right] D_{11}^{a}} \\
{\left[\mathbf{W}^{a}\right]=\left[\begin{array}{ccc}
0 & 0 & \rho_{13} \\
0 & 0 & \rho_{23} \\
-\rho_{13} & -\rho_{23} & 0
\end{array}\right] D_{11}^{a}}
\end{gathered}
$$

and they contain three so-called strain rate ratios:

$$
\rho_{22} \equiv \frac{D_{22}^{a}}{D_{11}^{a}}, \quad \rho_{13} \equiv \frac{D_{13}^{a}}{D_{11}^{a}}, \quad \rho_{23} \equiv \frac{D_{23}^{a}}{D_{11}^{a}}
$$

Some additional notes can be made on the equation (6) together with (8):

- Material incompressibility has been additionally assumed in equation (6), as $\operatorname{trace}\left(\mathbf{D}^{a}\right)=0$

- The component $D_{11}^{a}$ is explicitly written outside of the matrix representation of the tensor. By doing so, this scalar is considered as the measure of the rate of deformation. Only strain-rate insensitive material models are currently considered, so its value may actually be chosen freely. For convenience, $D_{11}^{a}$ is chosen so that:

$$
\left\|\mathbf{D}^{a}\right\|=1
$$

where $\|\mathbf{X}\|$ denotes the norm of $\mathbf{X}$. 
In the trivial case of $\rho_{13}=\rho_{23}=0$, these equations indicate that no shear is acting for any value of $\alpha$, i.e. on any plane containing the sheet normal direction. In all other cases however (i.e. with some kind of imposed TTS), the equations (10)-(11) show that there exists exactly one direction in the sheet plane onto which no through-thickness shear acts. Indeed, by imposing $D_{2^{\prime} 3}^{a}=0$ in the equation (11) for a particular choice of the angle $\alpha$, which is in this case called $\alpha_{T T S}$, it is found that:

$$
\tan \left(\alpha_{T T S}\right)=\frac{\rho_{23}}{\rho_{13}}
$$

This equation has always solutions if at least one of $\rho_{13}$ and $\rho_{23}$ is non-zero, and they are of the form $\alpha_{T T S} \pm n 180^{\circ}$ ( $n$ being an integer), so all solutions actually correspond to a single direction in the sheet plane. Mind that $\alpha_{T T S}$ actually refers to the perpendicular direction in the sheet plane, as it is an angle defined from the 1-axis to the 1'-axis, while $D_{2}^{a} 3$, i.e. the strain rate in the direction at an angle of $\alpha_{T T S}+90^{\circ}$ from the 1 -axis, was set to 0 in equation (11).

For this angle $\alpha_{T T S}$, the left-hand side of equation (10) is defined as $\rho_{T T S}$. It is the normalized through-thickness shear strain rate along the direction at the angle $\alpha_{T T S}$ from the major in-plane strain direction:

$$
\left.\rho_{T T S} \equiv \frac{D_{1^{\prime} 3}^{a}}{D_{11}^{a}}\right|_{\alpha=\alpha_{T T S}}=\cos \left(\alpha_{T T S}\right) \rho_{13}+\sin \left(\alpha_{T T S}\right) \rho_{23}
$$

After some elaboration, in which equation (12) is used, one finds:

$$
\rho_{T T S}^{2}=\rho_{13}^{2}+\rho_{23}^{2}
$$

The characterization of TTS with the ratios $\rho_{13}$ and $\rho_{23}$ can thus be converted to $\rho_{T T S}$ and $\alpha_{T T S}$ using the equations (12) and (14). The inverse relationship is given by: 


$$
\rho_{23}=\sin \left(\alpha_{T T S}\right) \rho_{T T S}
$$

Now suppose that one would subject a sheet to TTS $\left(\rho_{T T S}>0\right)$ in a certain sheet direction (and thus without TTS in the perpendicular sheet direction). Equation (15) can be used to calculate the TTS in some other sheet direction, such as the 1-direction lying at an angle $-\alpha_{T T S}$ from the original direction. Because of the form of the equation (15), it can be seen that through-thickness shear is maximal in the direction perpendicular to the direction in which TTS is equals zero.

The quantity $\rho_{T T S}$ is therefore called the magnitude of TTS. It is imposed along the socalled direction of TTS, which is described by $\alpha_{T T S}$. These two quantities can be more easily interpreted compared to a description of TTS using the ratios $\rho_{13}$ and $\rho_{23}$. For instance, one can imagine the angle $\alpha_{T T S}$ being determined by local friction with one or more forming tools.

A visualization of the TTS along all sheet directions can be made with a polar plot, of which Figure 2 gives an example. Any conceivable type of TTS looks like this one, except for possibly a rotation (different $\alpha_{T T S}$ ) and/or resizing (different $\rho_{T T S}$ ).

The imposed strain rate of the matrix, given in equation (6) can be rewritten using the equations (15)-(16):

$$
\left[\mathbf{D}^{a}\right]=\left[\begin{array}{ccc}
1 & 0 & \cos \left(\alpha_{T T S}\right) \rho_{T T S} \\
& \rho_{22} & \sin \left(\alpha_{T T S}\right) \rho_{T T S} \\
(\text { sym. }) & & -\left(1+\rho_{22}\right)
\end{array}\right] D_{11}^{a}
$$

in which the magnitude of the strain rate is defined by $D_{11}^{a}$, while the strain mode is determined by three independent variables: 
1. the in-plane strain rate mode $\rho_{22}$.

2. the direction of imposed TTS, characterized by $\alpha_{T T S}$, in the range $-180^{\circ} \leq \alpha_{T T S} \leq 180^{\circ}$.

3. the magnitude of imposed TTS $\rho_{T T S}$, in the range $\rho_{T T S} \geq 0$.

\subsection{Description of the rate of deformation in the groove}

In contrast to the velocity gradient of the matrix $\mathbf{L}^{a}$, the groove velocity gradient $\mathbf{L}^{b}$ is $a$ priori unknown. It is calculated through geometrical compatibility and force equilibrium with the surrounding matrix material, which is discussed in the sections 2.5-2.6, respectively. Because of this, it is preferred to express $\mathbf{L}^{b}$ in the groove reference frame $G$. It is then denoted $\left[\mathbf{L}^{b}\right]^{G}$ :

$$
\left[\mathbf{L}^{b}\right]^{G}=\left[\begin{array}{ccc}
L_{n n}^{b} & L_{n t}^{b} & L_{n 3}^{b} \\
L_{t n}^{b} & L_{t t}^{b} & L_{t 3}^{b} \\
L_{3 n}^{b} & L_{3 t}^{b} & L_{33}^{b}
\end{array}\right]
$$

It contains 9 unknown components. As an alternative representation, $\left[\mathbf{L}^{b}\right]^{G}$ can be decomposed into its symmetric and anti-symmetric parts. It is then expressed as follows by 6 independent components of the groove strain rate tensor $\left[\mathbf{D}^{b}\right]^{G}$ and 3 independent components of the rigid body rotation rate tensor $\left[\mathbf{W}^{b}\right]^{G}$ :

$$
\begin{aligned}
{\left[\mathbf{L}^{b}\right]^{G} } & =\left[\mathbf{D}^{b}\right]^{G}+\left[\mathbf{W}^{b}\right]^{G} \\
& =\left[\begin{array}{ccc}
D_{n n}^{b} & D_{n t}^{b} & D_{n 3}^{b} \\
D_{n t}^{b} & D_{t t}^{b} & D_{t 3}^{b} \\
D_{n 3}^{b} & D_{t 3}^{b} & D_{33}^{b}
\end{array}\right]+\left[\begin{array}{ccc}
0 & W_{n t}^{b} & W_{n 3}^{b} \\
-W_{n t}^{b} & 0 & W_{t 3}^{b} \\
-W_{n 3}^{b} & -W_{t 3}^{b} & 0
\end{array}\right]
\end{aligned}
$$


Imposing material incompressibility, the 9 unknown components are reduced to 8 , as:

$$
D_{33}^{b}=-D_{n n}^{b}-D_{t t}^{b}
$$

\subsection{The strain definition in matrix and groove}

The adopted measure to characterize the strain $\varepsilon^{x}$ (in either matrix or groove) is found in the global frame from the time integral of the respective strain rate tensor:

$$
\left[\boldsymbol{\varepsilon}^{x}\right]=\int_{0}^{T}\left[\mathbf{D}^{x}\right] d t
$$

in which $T$ is the total time. In component form, this is written as:

$$
\varepsilon_{i j}^{x}=\int_{0}^{T} D_{i j}^{x} d t
$$

The current thickness $\vartheta^{x}$ (of matrix or groove) is found from the normal strain in the thickness direction, as:

$$
\vartheta^{x}=\exp \left(\varepsilon_{33}^{x}\right) \vartheta_{0}^{x}
$$

The model solution progresses in small time increments of size $\Delta T$. During such small time increment, the expression of the velocity gradients of matrix and groove are assumed to be constant. For an increment, the corresponding incremental strain tensor $\left[\Delta \varepsilon^{x}\right]$ is then given by:

$$
\left[\Delta \mathbf{\varepsilon}^{x}\right]=\Delta T\left[\mathbf{D}^{x}\right]
$$




\subsection{Geometric compatibility}

In order to ensure geometric compatibility between the groove and surrounding matrix, the following five components of the expression of the groove velocity gradient in the groove reference frame $\left[\mathbf{L}^{b}\right]^{G}$ are forced to equal the respective values of the matrix velocity gradient $\left[\mathbf{L}^{a}\right]^{G}$ :

$$
\begin{aligned}
& L_{t t}^{b}=L_{t t}^{a} \\
& L_{n t}^{b}=L_{n t}^{a} \\
& L_{t 3}^{b}=L_{t 3}^{a} \\
& L_{3 t}^{b}=L_{3 t}^{a} \\
& L_{n 3}^{b}=L_{n 3}^{a}
\end{aligned}
$$

The other components of the groove velocity gradient are 'free', i.e. their value can differ from the corresponding matrix value. To motivate the particular choice that is made, all the compatibility conditions and freedoms of the groove are sketched in Figure 3.

Compared to a conventional MK model (i.e. without TTS), such as the one described in (Hiwatashi et al., 1998), it is seen that the compatibility conditions (27)-(29), all reflecting some kind of Through-Thickness Shear (TTS), have been added, while the conditions (25)(26) are the same as in (Hiwatashi et al., 1998).

Regarding the compatibility condition (28), it can be noted that actually

$$
L_{3 t}^{a}=0
$$


which follows from the restriction (4) on the form of the velocity gradient of the matrix $\mathbf{L}^{a}$. In other words, the apparent detachment of the groove reference frame from the sheet in the sketch of $L_{3 t}^{x}$ in the Figure 3(a), does not occur.

\subsection{Force equilibrium}

Consider two parallel and closely spaced planes perpendicular to the groove normal direction $n$, one of which lies in the matrix $a$, the other one in the groove $b$, as shown in Figure 4. In the stressed sheet, all material not confined between these planes is replaced with forces per unit groove length along the $t$-direction. They are noted in vector representation as $\mathbf{f}_{n}^{x}$, where the subscript $n$ refers to the plane onto which they act.

Force equilibrium between matrix and groove is imposed at the end of each small increment. It is expressed as:

$$
\mathbf{f}_{n}^{b}+\mathbf{f}_{n}^{a}=0
$$

Considering the homogeneous stress state within the matrix and within the groove, as well as their different thicknesses $\vartheta^{x}$, this can be rewritten in terms of traction stresses $\overrightarrow{\mathbf{t}}_{n}^{x}$ as:

$$
\vartheta^{b} \overrightarrow{\mathbf{t}}_{n}^{b}-\vartheta^{a} \overrightarrow{\mathbf{t}}_{n}^{a}=0
$$

which can be decomposed along the three directions of the groove reference frame:

$$
\begin{gathered}
\Delta f_{n n} \equiv \vartheta^{b} \sigma_{n n}^{b}-\vartheta^{a} \sigma_{n n}^{a}=0 \\
\Delta f_{t n} \equiv \vartheta^{b} \sigma_{t n}^{b}-\vartheta^{a} \sigma_{t n}^{a}=0
\end{gathered}
$$




$$
\Delta f_{3 n} \equiv \vartheta^{b} \sigma_{3 n}^{b}-\vartheta^{a} \sigma_{3 n}^{a}=0
$$

These functions (33)-(35) expressing force unbalance are denoted $\Delta f_{\text {in }}(i \in\{n, t, 3\})$. They contain components of the Cauchy stress tensor $\boldsymbol{\sigma}^{x}$ (superscript $x$ referring to matrix $a$ or groove $b$ ), represented in the groove reference frame $G$.

Comparing these force equilibrium equations (33)-(35) to the conventional MK model which does not incorporate Through-Thickness Shear (TTS), e.g. (Hiwatashi et al., 1998), it is seen that one new equation is added, namely equation (35). Indeed, a non-zero shear stress $\sigma_{3 n}$ is possible in the present model due to Through-Thickness Shear (TTS). In the conventional MK model in (Hiwatashi et al., 1998), equation (35) is implicitly satisfied, as $\sigma_{3 n}^{a}=\sigma_{3 n}^{b}=0$

As elaborated further in the section 2.10, the time integration of the constitutive law results in the deviatoric stress at the end of the increment $\mathbf{s}^{x}$ (cf. equation (54)), which is denoted $\left[\mathbf{s}^{x}\right]^{G}$ when expressed in the groove frame. To check the force equilibrium in the equations (33)-(35), the total stress in the groove frame $\left[\boldsymbol{\sigma}^{x}\right]^{G}$ is required. It is obtained from:

$$
\left[\boldsymbol{\sigma}^{x}\right]^{G}=\left[\mathbf{s}^{x}\right]^{G}+\sigma_{m}^{x}[\mathbf{I}]
$$

where $[\mathbf{I}]$ is the $3 * 3$ unit matrix. The (scalar) hydrostatic stress $\sigma_{m}^{x}$ is however unknown up to this point because of the assumption of material incompressibility (the elastic part is neglected), so every hydrostatic stress is allowed. 


\subsection{Solution procedure of an increment}

The numerical solution procedure described in this section is adopted for the formability predictions of section 3.3. It is pointed out that other numeric methods may be selected for the solution of the system of nonlinear force equilibrium equations (33)-(35).

Once a matrix strain path $\mathbf{D}^{a}(t)$ (cf. equation (6)) is chosen together with an initial groove angle $\Phi_{0}$, the set of 3 equilibrium equations (33)-(35) together with the set of 5 compatibility conditions (25)-(29) determine for each matrix strain increment the 8 unknown components $D_{n n}^{b}, D_{t t}^{b}, D_{n t}^{b}, D_{n 3}^{b}, D_{t 3}^{b}, W_{n t}^{b}, W_{n 3}^{b}$ and $W_{t 3}^{b}$ of the groove velocity gradient $\mathbf{L}^{b}$ as expressed in the equations (19)-(20). This set of 8 unknowns is firstly reduced to 3 by rewriting the 5 compatibility conditions (25)-(29) (which are linear equations) into:

$$
\begin{gathered}
D_{t t}^{b}=D_{t t}^{a} \\
W_{n t}^{b}=L_{n t}^{a}-D_{n t}^{b} \\
D_{t 3}^{b}=L_{t 3}^{a} / 2
\end{gathered}
$$




$$
\begin{gathered}
W_{t 3}^{b}=L_{t 3}^{a} / 2 \\
W_{n 3}^{b}=L_{n 3}^{a}-D_{n 3}^{b}
\end{gathered}
$$

Note that for equations (41)-(42), the property (30) has also been used.

Secondly, the 3 remaining unknowns $D_{n n}^{b}, D_{n t}^{b}$ and $D_{n 3}^{b}$ are determined from the 3 nonlinear force equilibrium equations (33)-(35) through minimization of the following function $F$ :

$$
F\left(D_{n n}^{b}, D_{n t}^{b}, D_{n 3}^{b}\right)=\left(\Delta f_{n n}\right)^{2}+\left(\Delta f_{t n}\right)^{2}+\left(\Delta f_{3 n}\right)^{2}
$$

which is imposed at the end of each matrix strain increment. The function $F$ depends on $D_{n n}^{b}, D_{n t}^{b}$ and $D_{n 3}^{b}$ in two ways. Firstly, the groove stress tensor components $\sigma_{n n}^{b}, \sigma_{t n}^{b}$ and $\sigma_{3 n}^{b}$ are functions of $\mathbf{D}^{b}$ (via the adopted constitutive law), and $\mathbf{D}^{b}$ depends on $D_{n n}^{b}, D_{n t}^{b}$ and $D_{n 3}^{b}$ only, after substitution of equations (20), (39) and (41) into $\left[\mathbf{D}^{b}\right]^{G}$ (cf. equation (19)). Secondly, $\vartheta^{b}$ is a function of $D_{n n}^{b}$ through equations (23), (22), (20) and (39).

Equation (44) is solved with the Fletcher-Reeves-Polak-Ribiere minimization algorithm, cf. (Press et al., 1992). The number of iterations per increment depends heavily on the chosen increment size. For the smallest chosen size of the matrix incremental strain, i.e. $\left\|\Delta \varepsilon^{a}\right\|=10^{-4}$, usually less than 10 iterations were required per increment. Larger increments require more iterations. The number of iterations also tends to increase as deformation progresses (i.e. as strain rate of groove deviates further from the strain rate in the matrix). After minimization, the value of $F$ is expected to be close to 0 , but not exactly equal to 0 because of the numerical minimization procedure used. 


\subsection{Necking Criteria}

Due to the initially slightly smaller thickness of the groove compared to the matrix, the strain will become more and more concentrated within the groove. Two criteria are adopted that define the onset of necking of the groove, for a certain assumed initial groove orientation $\Phi_{0}$. If either one of them is satisfied, the corresponding strain tensor in the matrix is called the necking strain, which is denoted by $\varepsilon^{*}\left(\Phi_{0}\right)$. Note that it generally depends on the choice of the initial groove orientation.

The first necking criterion reads:

$$
\left|D_{33}^{b}\right|>10\left|D_{33}^{a}\right|
$$

The factor of 10 is rather arbitrary and any other relatively large positive number can be used. The effect of this number on the matrix strain at necking is minimal, since the absolute value of the groove thickness strain rate $\left|D_{33}^{b}\right|$ increases very rapidly towards infinity at incipient necking.

The second adopted criterion states that necking of the groove occurs if there is no longer a solution of the groove deformation that satisfies the force equilibrium (31). In practice, this is the case when after the minimization of the function $F$ in equation (44), it is found that:

$$
F>\delta
$$

in which $\delta$ is a small positive value related to the numeric precision of the minimization algorithm. For the results presented in this paper, $\delta=10^{-10}$ was chosen.

The second necking criterion occurs more frequently for relative large matrix strain increments, e.g. $\left\|\Delta \varepsilon^{a}\right\|=10^{-2}$, while the first is more frequent for smaller strain increments, 
e.g. $\left\|\Delta \varepsilon^{a}\right\|=10^{-4}$. Nevertheless very similar necking strains are found for different sizes of the chosen matrix strain increment. This indicates that the chosen numeric solution procedure of an increment (section 2.7) is sufficiently accurate. The calculation time of necking strain (i.e. for a particular choice of initial groove orientation) is in the order of 1 minute on a $3 \mathrm{GHz}$ PC.

\subsection{Forming limit strain}

Up to this point, the MK model has considered one particular initial groove orientation $\Phi_{0}$. However, in view of the assumed inhomogeneity of all sheet, all in-plane directions are potential necking directions. Therefore, for each imposed strain path, the MK analysis is repeated for a large number of initial groove orientations $\Phi_{0}$ in the full range from $-90^{\circ}$ to $90^{\circ}$.

In the actual sheet, the first neck that develops (of all possible necking directions) marks the onset of sheet instability, i.e. the forming limit. Consequently, the major-in-plane forming limit strain $\varepsilon_{11}^{*}$ is found as the smallest of all major-in-plane necking strains $\varepsilon_{11}^{*}\left(\Phi_{0}\right)$ :

$$
\varepsilon_{11}^{*}=\underset{\Phi_{0}}{\operatorname{Min}}\left(\varepsilon_{11}^{*}\left(\Phi_{0}\right)\right)
$$

The major-in-plane forming limit strain $\varepsilon_{11}^{*}$ is thus independent of $\Phi_{0}$. The minor in-plane limit strain is for monotonic strain paths given by $\varepsilon_{22}^{*}=\rho_{22} \varepsilon_{11}^{*}$ (cf. equation (8)).

Obviously, in practice a finite number of initial groove directions has to be chosen. An interval of $1^{\circ}$ or $2^{\circ}$ has been found to be sufficient to accurately determine the minimum in equation (47). 


\subsection{Constitutive laws}

half this range, i.e. $\Phi_{0} \in\left[0^{\circ} ; 90\right]$, is taken into consideration in $\mathrm{MK}$ analyses from the literature. The reason is that when Through-Thickness Shear (TTS) is not considered, it is found that $\varepsilon^{*}\left(-\Phi_{0}\right) \approx \varepsilon^{*}\left(\Phi_{0}\right)$ (small differences are possible due to in-plane anisotropy). In this generalized MK model however, such (approximate) 'symmetry property' no longer holds, as the global reference frame need not coincide with the principal straining directions.

So far, the only assumptions for the material behaviour have been (i) material incompressibility (cf. equations (6) and (20)) and (ii) strain-rate insensitivity (cf. equation (9) ). In this section, the full description of the adopted material model is presented. It is a purely plastic material model featuring isotropic hardening and a plastic potential to describe anisotropic yielding.

As discussed in the introduction (section 1.2), the current MK analysis requires a local reference frame in which the constitutive law is expressed. In fact, the two zones of the model both have their own reference frame, as indicated in

. The definition of the local frame using the CSLVG-method is given in the next section 2.10.1.

The next step is to obtain the deviatoric stress mode from the local strain rate through the plastic potential (section 2.10.2). Combined with the hardening law, presented in section 2.10.3, this yields the deviatoric stress, which is in first instance expressed in the local frame. 
The constitutive equations are obviously valid for both matrix and groove zone. The superscript ' $x$ ', referring to either the matrix $a$ or groove $b$, is dropped in the equations in this section 2.10 to improve readability.

\subsubsection{The definition of the local frame}

The CSLVG-method makes use of the concept of a rotation tensor, which is denoted in the present work as $\mathbf{R}$ (in (de Montleau et al., 2008), the symbol $\boldsymbol{\rho}$ is used). It is in fact an (orthonormal) deformation gradient tensor that physically rotates material vectors along a local axis onto the respective global axis. The rotation tensor of the undeformed sheet, denoted $\mathbf{R}_{0}$, is known from the angle $\alpha_{R D}$ (cf.

(a)):

$$
\left[\mathbf{R}_{0}\right]=\left[\begin{array}{ccc}
\cos \left(\alpha_{R D}\right) & \sin \left(\alpha_{R D}\right) & 0 \\
-\sin \left(\alpha_{R D}\right) & \cos \left(\alpha_{R D}\right) & 0 \\
0 & 0 & 1
\end{array}\right]
$$

$\mathbf{R}_{0}$ is the same for matrix and groove. After some amount of deformation $\mathbf{R}$ is usually different for both zones (at least in the case that the strain history of matrix and groove differs).

The rotation tensor at the start and end of a certain increment is written as $\mathbf{R}_{\text {ini }}$ and $\mathbf{R}_{\text {end }}$, respectively. $\mathbf{R}_{\text {ini }}$ is known from the previous increment, while $\mathbf{R}_{\text {end }}$ is calculated by the CSLVG-method.

In the context of updated Lagrangian FE formulations for which the CSLVG-method has been developed, the incremental deformation gradient at an integration point $\Delta \mathbf{F}$ is assumed to be known. For a particular increment in time, $\Delta \mathbf{F}$ is the deformation gradient at the end of the increment with as a reference state the start of the increment. In the context of the current 
MK model framework, deformation is in first instance described by the velocity gradient $\mathbf{L}$,

in which $\mathbf{L}$ is the constant velocity gradient of the increment, with corresponding increment in time $\Delta T$. Note that this calculation requires the calculation of an exponent of a (generally) non-symmetric matrix, cf. equations (3) and (18), which is not obvious as pointed out in (de Montleau et al., 2008). The 'method 3' (Taylor series expansion method with scaling and squaring) of (Moler and Van Loan, 1978) has been adopted here.

As described in (de Montleau et al., 2008), the calculation of $\mathbf{R}_{\text {end }}$ is as follows:

$$
\begin{gathered}
{\left[\Delta \mathbf{F}^{*}\right]=[\Delta \mathbf{F}]\left[\mathbf{R}_{\text {ini }}\right]} \\
{\left[\Delta \mathbf{F}^{\prime}\right]=\left(\left[\Delta \mathbf{F}^{*}\right]^{T}\left[\Delta \mathbf{F}^{*}\right]\right)^{1 / 2}} \\
{\left[\mathbf{R}_{\text {end }}\right]=\left[\Delta \mathbf{F}^{*}\right]\left[\Delta \mathbf{F}^{\prime}\right]^{-1}}
\end{gathered}
$$

in which $\left[\Delta \mathbf{F}^{*}\right]$ is an intermediate tensor quantity and $\left[\Delta \mathbf{F}^{\prime}\right]$ is actually the local incremental deformation gradient, i.e. defined from coordinates in the local frame that rotates during the increment. Note that in equation (51), the square root of a symmetric matrix is required. The resulting matrix $\left[\Delta \mathbf{F}^{\prime}\right]$ is then also symmetric. The square root is computed in a very efficient way through the eigenvalue decomposition of symmetric matrices.

The local velocity gradient $\mathbf{L}^{\prime}$, i.e. the velocity gradient defined from coordinates in the local frame, is (i) constant during the increment and (ii) symmetric. This last property can be seen by the way it is calculated (de Montleau et al., 2008):

$$
\left[\mathbf{L}^{\prime}\right]=\frac{1}{2 \Delta T} \ln \left(\left[\Delta \mathbf{F}^{*}\right]^{T}\left[\Delta \mathbf{F}^{*}\right]\right)
$$


Note that here the natural logarithm of a symmetric matrix is required; the resulting matrix $\left[\mathbf{L}^{\prime}\right]$ is then also symmetric. The natural logarithm is computed in a very efficient way through the eigenvalue decomposition of symmetric matrices.

$\mathbf{L}^{\prime}$ being effectively symmetric, means that it equals the local strain rate, which is denoted $\mathbf{D}$ in the next section 2.10.2.

\subsubsection{Facet method to describe anisotropy}

As proposed in (Van Houtte et al., 2009), a plastic potential in strain-rate space $\psi(\mathbf{D})$ is defined in order to calculate the (deviatoric) stress tensor $\mathbf{s}$ from an imposed strain rate tensor D. For rate-insensitive materials, the plastic potential allows to calculate $\mathbf{s}$ as:

$$
s_{p}=\frac{\partial \psi(\mathbf{D})}{\partial D_{p}}
$$

Note the single index $p$ in $s_{p}$ and $D_{p}$ in equation (54): these deviatoric second-order tensors are represented by a 5-dimensional vector, as described in Appendix 1 of (Van Houtte et al., 2009).

The Facet method of (Van Houtte et al., 2009) for rate-insensitive materials is adopted, i.e. the plastic potential takes the form:

$$
\begin{gathered}
\psi(\mathbf{D})=\left(G_{n}\right)^{1 / n} \\
G_{n}=\sum_{\kappa=1}^{K} \lambda_{\kappa}\left(s_{\kappa p} D_{p}\right)^{n}
\end{gathered}
$$

It should be noted that the Einstein summation convention is followed for the index ' $p$ ' in equation (56). The integer $n \geq 2$ is called the order of the Facet expression. The parameters that describe the anisotropy are (i) the scalars $\lambda_{\kappa} \geq 0$ and (ii) the stress tensors $\mathbf{s}_{\kappa}$ (5dimensional vector representation). The integer $K$ is the number of terms in the Facet 
expression. For a low carbon steel and aluminium alloy, the section 3.2 describes a fitting procedure based on the Taylor-Bishop-Hill multilevel model.

\subsubsection{Strain hardening}

In the present work, isotropic hardening is assumed. For practical calculations of the plastic potential, it is in this case advantageous to work with the potential scaled by the equivalent yield stress $\sigma_{y}$ :

$$
\tilde{\psi}(\mathbf{D})=\frac{1}{\sigma_{y}} \psi(\mathbf{D})
$$

The advantage lies in the fact that $\tilde{\psi}(\mathbf{D})$ does not evolve during straining for an isotropically hardening material, so the expression of $\tilde{\psi}(\mathbf{D})$ at initial yield can be used throughout the subsequent deformation steps. Since $\psi$ equals the plastic work per unit volume (proof in (Van Houtte et al., 2009)), it follows from equation (57) that $\tilde{\psi}$ equals the equivalent strain rate $D_{e q}$ that is work-conjugate to $\sigma_{y}$ :

$$
D_{e q}=\tilde{\psi}(\mathbf{D})
$$

The purely isotropic hardening law of Swift is adopted. The equivalent yield stress $\sigma_{y}$ is thus obtained as follows:

$$
\sigma_{y}=K\left(\varepsilon_{0}+\varepsilon_{e q}\right)^{n}
$$

in which $K, \varepsilon_{0}$ and $n$ are the Swift hardening parameters, namely the strength coefficient, initial yield coefficient and hardening coefficient, respectively. The equivalent strain $\varepsilon_{e q}$ is given by the time integration of $D_{e q}$ :

$$
\varepsilon_{e q}=\int D_{e q} d t
$$


The deviatoric stress tensor $\mathbf{s}$ is required to impose force equilibrium (cf. section 2.6). However, in order to check the force equilibrium equations (33)-(35), first a tensor transformation from the local frame to the groove frame $G$ is required. This is most conveniently performed in two steps:

(i) Transformation from the respective local frame to the global one, using the respective rotation tensor $\mathbf{R}_{\text {end }}$, defined in equation (52). The subscript 'end' in this notation refers to the end of the increment.

(ii) Transformation from the global frame to the groove frame $G$, using a transformation matrix that is built from the groove angle $\Phi$ at the end of the increment, cf. equation (2).

The result is the deviatoric stress (of either the matrix or the groove) expressed in the groove frame $\left[\mathbf{s}^{x}\right]^{G}$, from which the total stress is calculated according to equation (36).

\subsection{Comparison to a different MK model with TTS}

As mentioned in the state-of-the-art (section 1.1), an alternative extension to the MK model has recently been proposed that accounts for TTS, i.e. the extension of (Allwood and Shouler, 2009). A discussion on two fundamental differences between the extension proposed presently and the one of Allwood, is given next.

Firstly, it is argued in the Allwood-MK model (Allwood and Shouler, 2009) that the traction stress on the sheet plane should be the same for both zones of the models. Three 'stress equilibrium equations' are thus introduced, one of which relates to the sheet normal stress, the other two to through-thickness shear stress. It implies that $\sigma_{n 3}^{b}=\sigma_{n 3}^{a}$, so force 
equilibrium between both zones is only partially fulfilled (compare to the force equilibrium equation (35) of the present extension). On the other hand, in the present extension only stress equilibrium of the sheet normal component is assumed (equation (37)). It is however not really considered to be a fundamental equation, but rather a necessity to obtain a unique solution for the mechanical response of the groove under the adopted material model (which assumes incompressibility and is therefore independent of hydrostatic stress). In fact, considering the deformation of a real sheet before the onset of strain localization, one can imagine that the macroscopic stress tensor is almost the same in different locations, but not exactly due to the intrinsic inhomogeneity. In terms of the MK model framework, a 'weak form' of stress equilibrium of the whole stress tensor should thus exist, at least before localization has taken place:

$$
\boldsymbol{\sigma}^{a} \approx \boldsymbol{\sigma}^{b}
$$

This weak stress equilibrium is already satisfied implicitly through the assumption of the initial imperfection $f_{0}$ that is close to, but smaller than 1 (' 1 ' meaning a perfectly homogeneous sheet). It is why in the extension presently proposed, an additional requirement for an exact equilibrium of some of the stress components is considered to be unnecessary (the exception is equation (37) for the reason explained above).

Secondly, the geometric compatibility between both zones is in the Allwood extension only partially imposed. Only one of the five compatibility equations (25)-(29) of the present extension is adopted, i.e. the one related to the normal strain along the groove direction (it corresponds to the equation (25)). It should be noted that in the extension presently proposed, the groove in the MK model is considered to be a physical part of the sheet: it is the material of the localized neck before the onset of necking. Geometric compatibility should thus be enforced 'as much as possible', but still allowing for the localization of strain within the neck. On this basis, the conditions (25)-(29) have been selected. Also, the extension of 
Allwood does not consider rigid body spin. Through-thickness shearing during deformation bares resemblance to a simple shear deformation (with the shearing direction in the sheet plane and the shear normal direction along the sheet normal), implying that rigid body spin is non-negligible. The use of the velocity gradient, adopted in the presently proposed extension, allows the more complete description of the instantaneous change in deformation, since it also comprises the rigid body spin besides the strain rate.

It is clear from the analysis of this section that the introduction of TTS in the MK model framework can be based on different assumptions. Arguments were given why the MK extension presently proposed does not assume the same constraints between matrix and groove (i.e. stress/force equilibrium and geometric compatibility) as the extension proposed by Allwood in (Allwood and Shouler, 2009). Future Finite Element studies, in which a groove is introduced in a sheet subjected to in-plane deformation and through-thickness shearing, could show to be very useful in assessing the most reasonable constraints for a MK model featuring TTS. 


\section{Model application}

\subsection{Material description}

Two commercially available materials have been selected. The first one is the aluminium alloy sheet AA3103 of $1.5 \mathrm{~mm}$ thickness in the fully annealed state. Its main alloying elements $\mathrm{Mn}$ and Fe form second phase intermetallic particles with Al. The second sheet is the low carbon steel named DC01, with a thickness of $1.0 \mathrm{~mm}$. It contains about $0.05 \% \mathrm{C}$.

Figure 5 presents the hardening behaviour of both materials observed in a representative tensile test along the rolling direction RD until the onset of diffuse necking. It is seen in Figure 5 that in this strain region the hardening can be approximated well with the Swift hardening law. The parameters of the Swift approximation in Figure 5 can be found in Table 1.

To determine the initial texture of both materials, texture measurements by X-ray diffraction were performed at half the sheet thickness. For each sheet, four incomplete pole figures were measured, i.e. (111)-, (200)-, (220)- and (311)-pole figures for AA3103 and (110)-, (200)-, (211)- and (311)-pole figures for DC01. The orientation distribution function (ODF) was calculated from the measured incomplete pole figures using the MTM-FHM software (Van Houtte, 2004). The Bunge convention for Euler angles is adopted (Bunge, 1982). The chosen maximal order of the series expansion Lmax is 22. Orthorhombic sample symmetry was imposed in the ODF calculation, since this symmetry could be seen in the measured pole figures of these rolled sheets.

Selected ODF sections that contain the most relevant texture components are given in Figure 6 and Figure 7, for the AA3103 and DC01 sheets, respectively. 
fibre contribute to in-plane anisotropy, which is generally undesirable. From the texture analysis of the particular sheet, a high average R-value is expected and a moderate in-plane anisotropy.

\subsection{Facet description based on the Taylor model}

As a first application of the extended MK model with the Facet method to describe material anisotropy (Van Houtte et al., 2009), the full-constraints Taylor multilevel model (Taylor, 1938) is chosen. It is a fairly simple multilevel model, as all grain interactions are neglected. It assumes that the strain of each grain equals the one imposed onto the whole polycrystal. More advanced multilevel models, like self-consistent models, GIA model and advanced LAMEL model (all discussed in (Van Houtte et al., 2005)), do take grain interaction into account and may therefore in future research produce a more accurate description of anisotropy.

The fitting procedure of both textures is performed in strain rate space as follows:

(i) The order $n=8$ is chosen for the Facet expression, as well as $K=2482$ strain modes, which cover the whole 5-dimensional stress space with an angular resolution of about $17^{\circ}$. For $n=8$, this value of $K$ is recommended in (Yerra, 2010).

(ii) For each of these strain modes $\mathbf{D}_{\kappa}(1 \leq \kappa \leq K)$, the corresponding stress mode $\mathbf{s}_{\kappa}$ and average Taylor factor $\bar{M}_{\kappa}$ are calculated by applying the conventional Taylor-Bishop-Hill model on a discrete set of 5000 orientations that describe the complete ODF of the respective texture. For the aluminium alloy, the 12 slip systems $\{111\}<110\rangle$ are considered in the multilevel model. For the ferritic steel, the 24 slip systems $\{110\}+\{112\}<111>$ are chosen. In both 
(iii) The remaining parameters $\lambda_{\kappa}$ of equation (56) are iteratively calculated according to the procedure proposed in (Van Houtte et al., 2009), until a converged approximation of the average Taylor factors is obtained.

A verification of the quality of the Facet method is made using the MTM-FHM software (Van Houtte, 2004). A first verification, cf. Figure 8, is given in terms of the $q$-values, i.e. the ratio of width contraction to longitudinal elongation under uniaxial tension (Bunge, 1970). The uniaxial tension direction may be varied within the sheet plane, which is characterized by the angle towards the rolling direction RD. The trends in $q$-values are seen to be well reproduced for both materials. Note that most of the Taylor results in this figure have actually not been used in the identification procedure.

A second verification is made in terms of the yield locus derived from the Facet potential. Figure 9 shows the $\sigma_{11}-\sigma_{22}$-section, in which the 1- and 2-directions correspond to the rolling direction RD and transverse direction $\mathrm{TD}$, respectively. It is compared to the socalled geometric method, which constructs an estimate of the yield locus based on a limited number of Taylor simulations ((Van Houtte et al., 1989)). In Figure 8, it is seen that the Facet method reproduces the yield locus very well for both materials. The AA3103 yield locus section is clearly less rounded than the low carbon steel DCO1. This is typical for aluminium alloys, and is related to the number of slip planes, which is only 12 for fcc materials like aluminium alloys, while it is 24 for bcc materials like ferritic steels. 
Since the stress modes encountered in the extended MK model are not limited to this section, additional sections are presented in the Figure 10. While the horizontal axis corresponds to the normal stress in the rolling direction $\sigma_{11}$, the vertical axis is one of the three following shear stress components: $\sigma_{23}$ (through-thickness shear stress along the transverse direction TD), $\sigma_{13}$ (through-thickness shear stress along the rolling direction RD), or $\sigma_{12}$ (in-plane shear stress). The three sections are superimposed to show the anisotropy in terms of shear stresses. The trends of the Taylor model yield locus (geometric method) are captured by the Facet method. For AA3103, $\sigma_{23}$ is clearly different from both other shear stresses. Whereas for DC01 the differences between the three yield loci sections is quite small for relative small shear strain, the curve with the in-plane shear stress $\sigma_{12}$ clearly diverges from both other curves towards the yield point under pure shear (on the vertical axis).

\subsection{Formability predictions}

In all forming limit calculations presented hereafter, an initial imperfection $f_{0}=0.998$ is used, which is a realistic value for many materials. The choice of a fixed value of $f_{0}$ is justified by the fact that the main focus is the effect of TTS on forming limit predictions. It is however acknowledged that a fully quantitative formability prediction would require a fitting of the most suitable initial imperfection parameter $f_{0}$ (defined in equation (1)) for each particular material sheet. Predictions obtained with the use of the Facet plastic potential are compared to those that adopt a von Mises yield locus (cf. (Eyckens et al., 2009)) and the same isotropic Swift hardening law. 


\subsubsection{The forming limit without through-thickness shear}

Figure 11 shows the FLCs for both materials without TTS $\left(\rho_{T T S}=0\right)$ for the anisotropic yield locus derived from the Facet plastic potential, as well as the isotropic von Mises yield locus. For the former, three different directions of the rolling direction RD with respect to the major in-plane strain direction are considered, as shown in the legend. It is apparent that for both materials, the Facet yield locus give very different formability predictions in the righthand side of the FLD. Referring to Figure 9, the difference between both materials can be related to the difference in their yield loci in the region between the equibiaxial and plane strain yield point. More in particular, the AA3103 has the smallest formability because the ratio of plane strain yield stress over equibiaxial yield stress is the smallest, and consequently the delay in the onset of necking due to the strain mode change of the groove towards plane strain, is very limited (cf. the discussion in section 4.2 of (Eyckens et al., 2009)). On the other hand, the 'more rounded' yield locus of the DC01 has a formability comparable to von Mises, while their respective yield loci are also similar.

The left-hand side of the FLD is seen to be practically independent of the adopted yield locus. It is interesting to make the comparison with the bifurcation analysis of (Hill, 1952), which gives a limit strain of:

$$
\varepsilon_{11}^{*}=\frac{n}{1+\rho_{22}} \quad \text { for } \rho_{22} \leq 0 \quad\left(\rho_{T T S}=0\right)
$$

This equation is derived for a material that has Swift hardening as in equation (59), but with $\varepsilon_{0}=0$, and that has a normal anisotropy. The forming limit derived from it is however independent of the normal anisotropy parameter. In the FLD, it corresponds to a line with a slope of $-45^{\circ}$ that intersects with the vertical axis $\left(\rho_{22}=0\right)$ at $\varepsilon_{11}^{*}=n$. Although the approaches of MK and Hill are very different, the formability predictions are seen to compare 
very well in this region of the FLD. They correspond to the following formability criterion in terms of the thickness strain:

$$
\varepsilon_{33}^{*}=-n \quad \text { for } \rho_{22} \leq 0 \quad\left(\rho_{\text {TTS }}=0\right)
$$

In (Chan et al., 1984), this is also discussed as well as experimentally validated.

A last observation from Figure 11 is that the sheet orientation with respect to the principal in-plane straining directions has for both materials only a small effect on the forming limit. The near-symmetry around a line at $45^{\circ}$ in the Facet yield locus sections in Figure 9 is directly reflected in the nearly identical FLCs between the curves with $\alpha_{R D}=0^{\circ}$ and $\alpha_{R D}=90^{\circ}$ in Figure 11.

\subsubsection{The effect of through-thickness shear on the forming limit}

The effect of Through-Thickness Shear (TTS) along the minor in-plane strain direction $\left(\alpha_{T T S}=90^{\circ}\right)$ is seen in Figure 12. It gives formability predictions obtained by using the Facet method, but also those obtained with the von Mises yield locus. The effect of anisotropy can be seen from their comparison. The strain paths with TTS have been analyzed in the sections 4.3-4.4 of (Eyckens et al., 2009), for a von Mises material with $\rho_{22}=0$ or $\rho_{22}<0$, respectively. The significant increase in formability that can be seen has been explained there by a change of the groove strain mode. It is thus expected that the local shape of the Facet yield locus, which is obviously different for both materials, influences the prediction. This is indeed seen in Figure 12. For instance, in case $\rho_{22}=0$, the forming limit obtained by using the Facet yield locus of AA3103 is higher than for von Mises, while for DC01, it is somewhat lower. Nonetheless, the same qualitative trend of increased formability is seen for all yield loci, since the underlying mechanism of groove strain mode change is in any case the same. Whereas the left-hand side of the FLCs in Figure 12 are only sensitive to 
the anisotropy related to TTS, the right-hand side is also sensitive to in-plane anisotropy, which can also be seen in Figure 11.

In the Figure 12, the rolling direction RD is aligned with the major in-plane strain direction, i.e. $\alpha_{R D}=0^{\circ}$. The influence of a different orientation on the formability of the anisotropic Facet yield loci is presented in the Figure 13 for $\alpha_{T T S}=90^{\circ}$. In both figures, the magnitude of TTS is taken to be $\rho_{T T S}=0.4$. Without TTS, the effect of $\alpha_{R D}$ on the forming limit is negligible for both materials (Figure 11). For strain paths containing TTS however, its influence on formability is seen to be quite significant for the AA3103 anisotropic material in case $\alpha_{T T S}=90^{\circ}$ (top FLD in Figure 13). A similar effect of anisotropy is less pronounced for the DC01 (bottom FLD in Figure 13).

Note that the difference between the yield loci sections marked with the diamonds and the squares in Figure 10 (i.e. the ones with non-zero through-thickness shear stress) is clearly larger for the AA3103 than for the DC01. It indicates that the AA3103 yield locus is more anisotropic in terms of these shear stresses. This explains why the forming limits in Figure 13 are more sensitive to $\alpha_{R D}$ for the AA3103 compared to the DC01.

\subsubsection{The influence of the direction of through-thickness shear on the forming limit}

The assumptions which have been made regarding force equilibrium and geometric compatibility in the present model (cf. sections 2.5-2.6), lead to a tremendous influence of the 
direction of the applied TTS on formability. To illustrate this point, Figure 14 shows the FLCs for a range of $\alpha_{T T S}$ with $\rho_{T T S}=0.4$.

It is seen that, especially for $\rho_{22} \leq 0$, TTS along the minor in-plane strain direction (i.e. $\alpha_{T T S}=90^{\circ}$ ) has the most beneficial effect on formability, but the extent of it depends on the anisotropic properties. In contrast to this, the direction of TTS has no effect at all in the MK model extension of (Allwood and Shouler, 2009). As stated there, this is a direct result of the choices of stress and force equilibrium equations, which are such that the stress and strain evolution within the groove does not depend on the direction of TTS. However, the force equilibrium equation (35) of the current extension, which is related to the shear stress between the matrix and groove in the thickness direction and which is not assumed in the Allwood-extension, does introduce a dependency of formability on the direction of TTS. 


\section{Conclusion}

This paper presents an extension to the Marciniak-Kuczynski (MK) model framework, which aims to predict the onset of localized necking during forming of sheet metal. This extension allows taking Through-Thickness Shear (TTS) during in-plane deformation explicitly into account. In essence, it is achieved through the addition of new geometric compatibility conditions and force equilibrium equations, which together describe the mechanical coupling between both zones in the MK framework. Arguments are provided why the present extension is more physically based compared to a recently proposed alternative approach ((Allwood and Shouler, 2009)).

Tensor analysis leads to conclude that in general, TTS can be associated to a particular direction in the sheet plane in which it is extreme. A deformation mode thus consists in general of only three independent variables, i.e. the in-plane strain mode, the in-plane direction of TTS, and the magnitude of TTS, whereas traditional MK models only consider the in-plane strain mode. For anisotropic sheet, an additional parameter has been included that expresses the initial sheet orientation.

The combination of TTS with anisotropic plastic behaviour leads to two additional issues that are usually not encountered in the MK model framework. Firstly, the local material frame proposed in (de Montleau et al., 2008) is incorporated, so that rotation of anisotropic properties is considered during shearing. Secondly, to describe anisotropic yielding for strain modes that have non-zero TTS components, it is proposed to use the Facet method, proposed in (Van Houtte et al., 2009), to construct the plastic potential. It considers strain modes that cannot be tested experimentally, through 'virtual testing' that is performed in an underlying multilevel model. 
From the textures of a Mn-Fe alloyed aluminium sheet and a low carbon steel sheet, the Facet potential is obtained from virtual tests of the Taylor-Bishop-Hill multilevel model, revealing out-of-plane anisotropy that cannot be obtained by mechanical tests. The formability predictions show that TTS can increase formability. It is seen that the shape of the anisotropic yield locus influences the formability predictions for monotonic strain paths that include TTS. For the investigated aluminium texture, formability is higher compared to the isotropic von Mises yield locus, while this is not the case for the investigated low carbon steel texture. The sheet orientation has a limited influence on the beneficial effects of TTS on the forming limit. Formability predictions are seen to be greatly affected by the direction of applied TTS with respect to the major in-plane strain direction. This last result is in contrast to the results obtained with the model of (Allwood and Shouler, 2009), in which the direction of TTS does not influence formability. Experimental or Finite Element studies that would (dis-)confirm the importance of the direction of TTS on formability is however, to the authors' knowledge, not presently available, and offers a challenge for future investigations. 


\section{Acknowledgements}

The authors gratefully acknowledge the financial support from the Institute for the Promotion of Innovation by Science and Technology in Flanders (IWT) and from the Interuniversity Attraction Poles Program from the Belgian State through the Belgian Science Policy agency, contract IAP6/24. 


\section{References}

Ahmadi, S., Eivani, A.R., Akbarzadeh, A., 2009. An experimental and theoretical study on the prediction of forming limit diagrams using new BBC yield criteria and $\mathrm{M}-\mathrm{K}$ analysis. Computational Materials Science 44, 1272-1280.

Allwood, J.M., Shouler, D.R., 2009. Generalised forming limit diagrams showing increased forming limits with non-planar stress states. Int. J. Plasticity 25, 1207-1230.

Aretz, H., 2007. Numerical analysis of diffuse and localized necking in orthotropic sheet metals. Int. J. Plasticity 23, 798-840.

Aretz, H., 2008. A simple isotropic-distortional hardening model and its application in elastic-plastic analysis of localized necking in orthotropic sheet metals. Int. J. Plasticity 24 , 1457-1480.

Assempour, A., Nejadkhaki, H.K., Hashemi, R., 2010. Forming limit diagrams with the existence of through-thickness normal stress. Computational Materials Science 48, 504-508.

Banabic, D., Soare, S., 2008. On the effect of the normal pressure upon the forming limit strains, in: Hora, P. (Ed.), Proceedings of the 7th International Conference and Workshop on Numerical Simulation of 3D Sheet Metal Forming Processes, Interlaken, Switserland, pp. 199-204.

Barata da Rocha, A., Santos, A.D., Teixeira, P., Butuc, M.C., 2009. Analysis of plastic flow localization under strain paths changes and its coupling with finite element simulation in sheet metal forming. Journal of Materials Processing Technology 209, 5097-5109.

Boudeau, N., Gelin, J.C., Salhi, S., 1998. Computational prediction of the localized necking in sheet forming based on microstructural material aspects. Computational Materials Science 11, 45-64.

Bressan, J.D., Williams, J.A., 1983. The use of a shear instability criterion to predict local necking in sheet metal deformation. Int. J. Mech. Sci. 25, 155-168.

Bunge, H.J., 1970. Some applications of the Taylor theory of polycrystal plasticity. Kristall und Technik 5, 145-175.

Bunge, H.J., 1982. Texture Analysis in Materials Science: Mathematical Methods. Butterworth, London.

Cahn, R.W., 1991. Processing of Metals and Alloys (Series: Materials Science and Technology, A Comprehensive Treatment). VCH, Weinheim.

Chan, K.S., Koss, D.A., Ghosh, A.K., 1984. Localized necking of sheet at negative minor strains. Metall. Trans. A 15A, 323-329.

Charca Ramos, G., Stout, M., Bolmaro, R.E., Signorelli, J.W., Serenelli, M., Bertinetti, M.A., Turner, P., In Press. Study of a drawing-quality sheet steel. II: Forming-limit curves by experiments and micromechanical simulations. Int. J. Solids Struct., In Press, Corrected Proof.

Chung, K., Ahn, K., Yoo, D.-H., Chung, K.-H., Seo, M.-H., Park, S.-H., In Press. Formability of TWIP (twinning induced plasticity) automotive sheets. Int. J. Plasticity, In Press, Corrected Proof.

de Montleau, P., Habraken, A.M., Duchêne, L., 2008. A new finite element integration scheme. Application to a simple shear test of anisotropic material. International Journal for Numerical Methods in Engineering 73, 1395-1412.

Duchêne, L., Lelotte, T., Flores, P., Bouvier, S., Habraken, A.M., 2008. Rotation of axes for anisotropic metal in FEM simulations. Int. J. Plasticity 24, 397-427.

Eyckens, P., Van Bael, A., Van Houtte, P., 2008. An Extended Marciniak-Kuczynski forming limit model to assess the influence of Through-Thickness Shear on Formability, in: Hora, P. 
(Ed.), Proceedings of the 7th International Conference and Workshop on Numerical

Eyckens, P., Van Bael, A., Van Houtte, P., 2009. Marciniak-Kuczynski type modelling of the effect of Through-Thickness Shear on the forming limits of sheet metal. Int. J. Plasticity 25, 2249-2268.

Hashiguchi, K., Protasov, A., 2004. Localized necking analysis by the subloading surface model with tangential-strain rate and anisotropy. Int. J. Plasticity 20, 1909-1930.

Hill, R., 1952. On Discontinuous Plastic States, with Special Reference to Localized Necking in Thin Sheets. J. Mech. Phys. Solids 1, 19-30.

Hiwatashi, S., Van Bael, A., Van Houtte, P., Teodosiu, C., 1998. Prediction of forming limit strains under strain-path changes: Application of an anisotropic model based on texture and dislocation structure. Int. J. Plasticity 14, 647-669.

Hughes, T.J.R., Winget, J., 1980. Finite Rotation Effects in Numerical Integration of Rate Constitutive Equations Arising in Large-deformation Analysis. International Journal of Numerical Methods in Engineering 15, 1862-1867.

Lévesque, J., Inal, K., Neale, K.W., Mishra, R.K., 2010. Numerical modeling of formability of extruded magnesium alloy tubes. Int. J. Plasticity 26, 65-83.

Liu, W.C., Zhai, T., Morris, J.G., 2004. Texture evolution of continuous cast and direct chill cast AA 3003 aluminum alloys during cold rolling. Scripta Materialia 51, 83-88.

Makkouk, R., Bourgeois, N., Serri, J., Bolle, B., Martiny, M., Teaca, M., Ferron, G., 2008. Experimental and theoretical analysis of the limits to ductility of type 304 stainless steel sheet. European Journal of Mechanics - A/Solids 27, 181-194.

Marciniak, Z., Kuczynski, K., 1967. Limit Strains in the Processes of Stretch-forming Sheet Metal. Int. J. Mech. Sci. 9, 609-620.

Moler, C.B., Van Loan, C.F., 1978. Nineteen Dubious Ways to Compute the Exponential of a Matrix. SIAM Review 20, 801-836.

Neil, J.C., Agnew, S.R., 2009. Crystal plasticity-based forming limit prediction for non-cubic metals: Application to Mg alloy AZ31B. Int. J. Plasticity 25, 379-398.

Press, W.H., Flannery, B.P., Teukolsky, S.A., Vetterling, W.T., 1992. Numerical Recipes in FORTRAN 77, 2nd ed. ed. Cambridge University Press.

Signorelli, J.W., Bertinetti, M.A., Turner, P.A., 2009. Predictions of forming limit diagrams using a rate-dependent polycrystal self-consistent plasticity model. Int. J. Plasticity 25, 1-25. Taylor, G.I., 1938. Plastic strain in metals. Journal institute of metals 62, 307-324.

Van Houtte, P., 2001. Fast calculation of average Taylor factors and Mandel spins for all possible strain modes. Int. J. Plasticity 17, 807-818.

Van Houtte, P., 2004. The MTM-FHM software system, Version 2.

Van Houtte, P., Li, S., Seefeldt, M., Delannay, L., 2005. Deformation texture prediction: from the Taylor model to the advanced Lamel model. Int. J. Plasticity 21, 589-624.

Van Houtte, P., Mols, K., Van Bael, A., Aernoudt, E., 1989. Application of yield loci calculated from texture data. Textures and Microstructures 11, 23-39.

Van Houtte, P., Yerra, S.K., Van Bael, A., 2009. The Facet method: A hierarchical multilevel modelling scheme for anisotropic convex plastic potentials. Int. J. Plasticity 25, 332-360.

Wu, P.D., Embury, J.D., Lloyd, D.J., Huang, Y., Neale, K.W., 2009. Effects of superimposed hydrostatic pressure on sheet metal formability. Int. J. Plasticity 25, 1711-1725.

Yerra, S., 2010. Ductile damage in single crysytals and anisotropic polycrystals (chapter 4), MTM. Katholieke Universiteit Leuven, PhD thesis.

Yoshida, K., Suzuki, N., 2008. Forming limit stresses predicted by phenomenological plasticity theories with anisotropic work-hardening behavior. Int. J. Plasticity 24, 118-139.

Yoshida, K., Tadano, Y., Kuroda, M., 2009. Improvement in formability of aluminum alloy sheet by enhancing geometrical hardening. Computational Materials Science 46, 459-468. 


\section{$\underline{\text { Table and figure captions }}$}

Figure 1 Scheme of the extended MK model, showing the matrix $a$ and the groove $b$ (in a lighter shade) in (a) the undeformed state, and (b) a deformed state that includes Through-Thickness Shear (TTS). The global reference frame has the 1- and 2-axes (in the sheet plane) and the 3-axis (along the sheet normal). The groove reference frame $G$ has the $n$-, $t$ - and 3- axes. The local frame of the matrix $a$ has the $1^{\mathrm{A}}$-, $2^{\mathrm{A}}$ - and $3^{\mathrm{A}}$-axes. The local frame of the groove $b$ has the $1^{\mathrm{B}}$-, $2^{\mathrm{B}}$ - and $3^{\mathrm{B}}$-axes. By convention, the angles $\Phi_{0}, \Phi$ and $\alpha_{R D}$ have a positive sign as drawn here.

Figure 2 Polar plot of normalized Through-Thickness Shear (TTS) in all sheet directions. The 1- and 2 -axis correspond to the major and minor in-plane strain directions, respectively. TTS of magnitude $\rho_{T T S}>0$ is imposed in the direction defined by $\alpha_{T T S}$. In the perpendicular direction, at $\alpha_{T T S} \pm 90^{\circ}$, there acts no TTS. The complete polar plot shows two circular lobes of opposite sign.

Figure 3 Visualization of (a) the five geometrical compatibility conditions of the groove velocity gradient and (b) the remaining free components.

Figure 4 Force equilibrium between matrix $a$ and groove $b$, viewed along (a) the sheet normal direction 3 and (b) along the groove direction $t$.

Figure 5 Hardening curves of tensile tests along the rolling direction RD, and the Swift hardening law (cf. equation (59)) with the parameters of Table 1.

Table 1 Swift hardening parameters.

Figure 6 The $\varphi_{2}=45^{\circ}, \varphi_{2}=65^{\circ}$ and $\varphi_{2}=90^{\circ}$ sections of the ODF of AA3103-O $(1.5 \mathrm{~mm}$ thickness) with the location of the most important texture components of aluminium alloys. Maximum value of ODF: 10.85 . Texture index: 2.0 .

Figure 7 The $\varphi_{1}=0^{\circ}, \varphi_{2}=45^{\circ}$ and $\varphi_{1}=90^{\circ}$ sections of the ODF of DC01 (1.0 mm thickness) with the location of the most important texture components of ferritic steels. Maximum value of ODF: 13.26. Texture index: 4.8.

Figure $8 q$-value as a function of the angle from the tensile direction to the rolling direction RD. 
Figure $9 \sigma_{11}-\sigma_{22}$ yield loci section, normalized by $\tau^{c}$. 1//RD, 2//TD.

Figure $10 \sigma_{11}-\sigma_{i j}$ yield loci sections, normalized by $\tau^{c}$. 1//RD, 2//TD, 3//ND.

Figure 11 Forming Limit Curves for the AA3103 and DC01 without TTS $\left(\rho_{T T S}=0\right)$, with three different angles between the major in-plane strain direction and the rolling direction.

Figure 12 Forming Limit Curves for the AA3103 and DC01 with three different amounts of TTS along the minor in-plane straining direction $\left(\alpha_{T T S}=90^{\circ}\right), \alpha_{R D}=0^{\circ}$.

Figure 13 Forming Limit Curves for the AA3103 and DC01 with TTS along the minor in-plane straining direction $\left(\rho_{T T S}=0.4 ; \alpha_{T T S}=90^{\circ}\right)$ and three different angles between the major in-plane strain direction and the rolling direction.

Figure 14 Forming Limit Curves for the AA3103 and DC01 with TTS along 7 different directions $\left(\rho_{T T S}=0.4\right) . \alpha_{R D}=0^{\circ}$. 

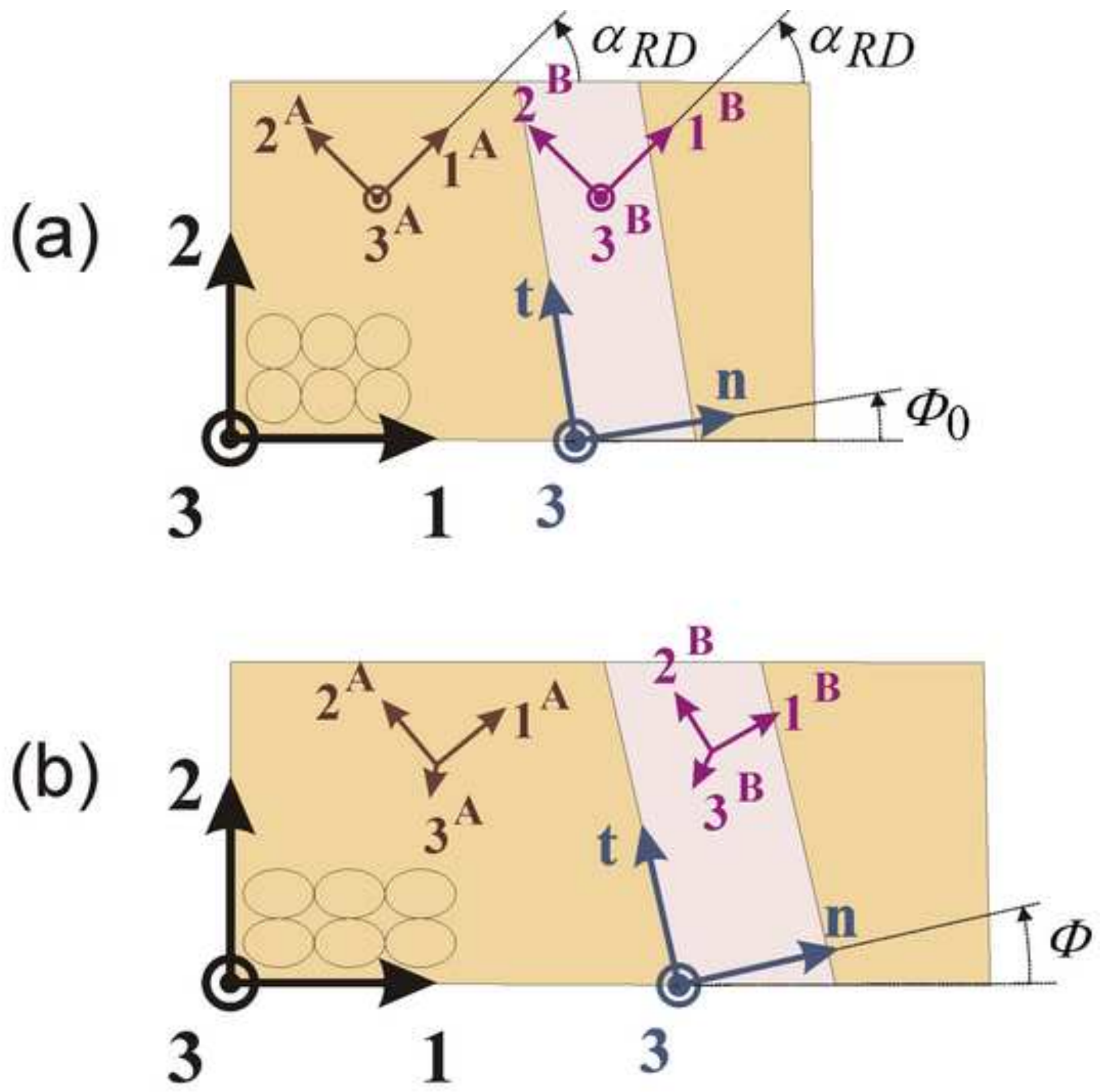


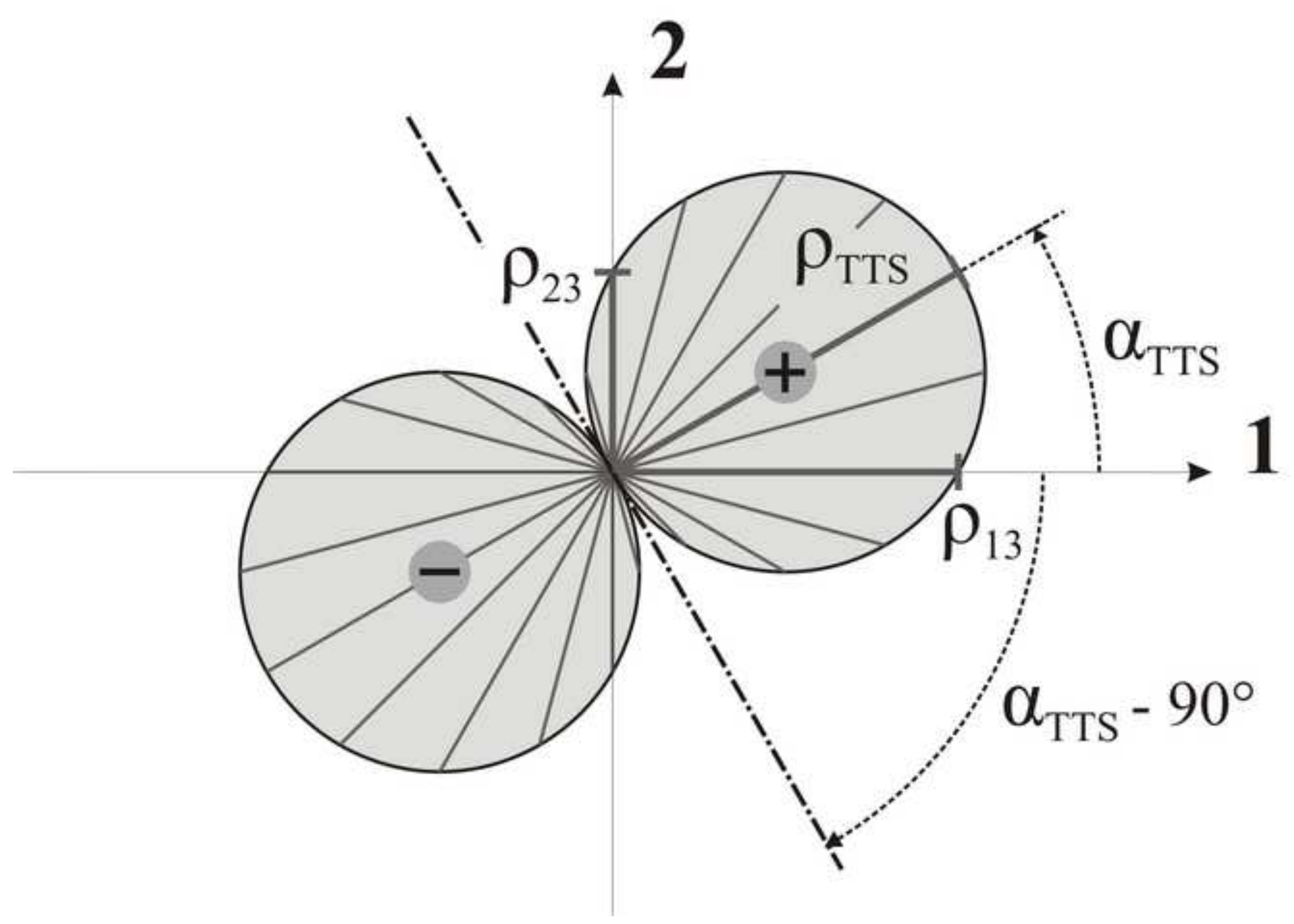


Click here to download high resolution image
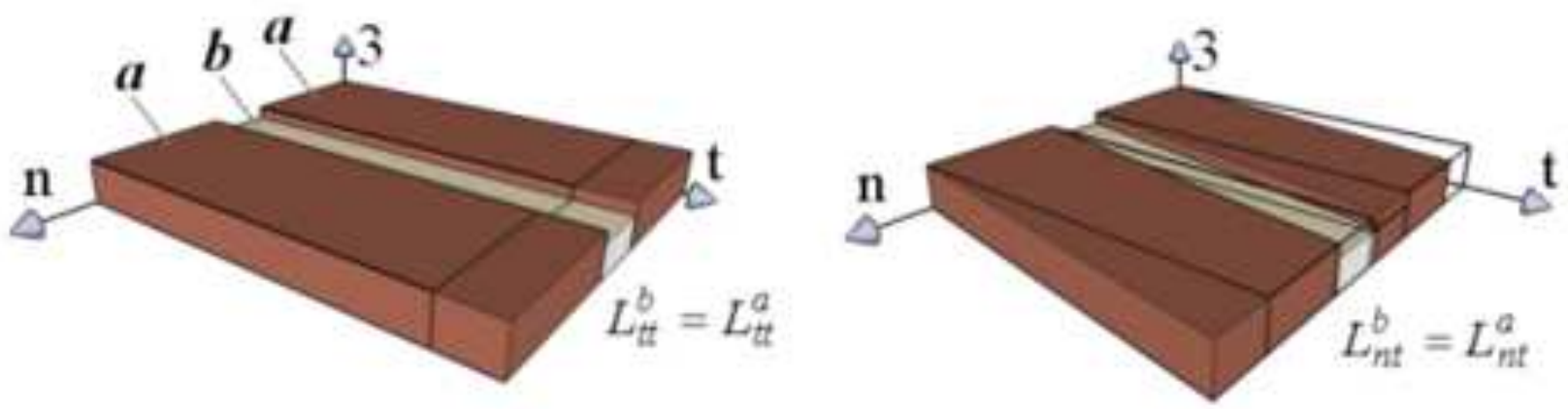

(a)
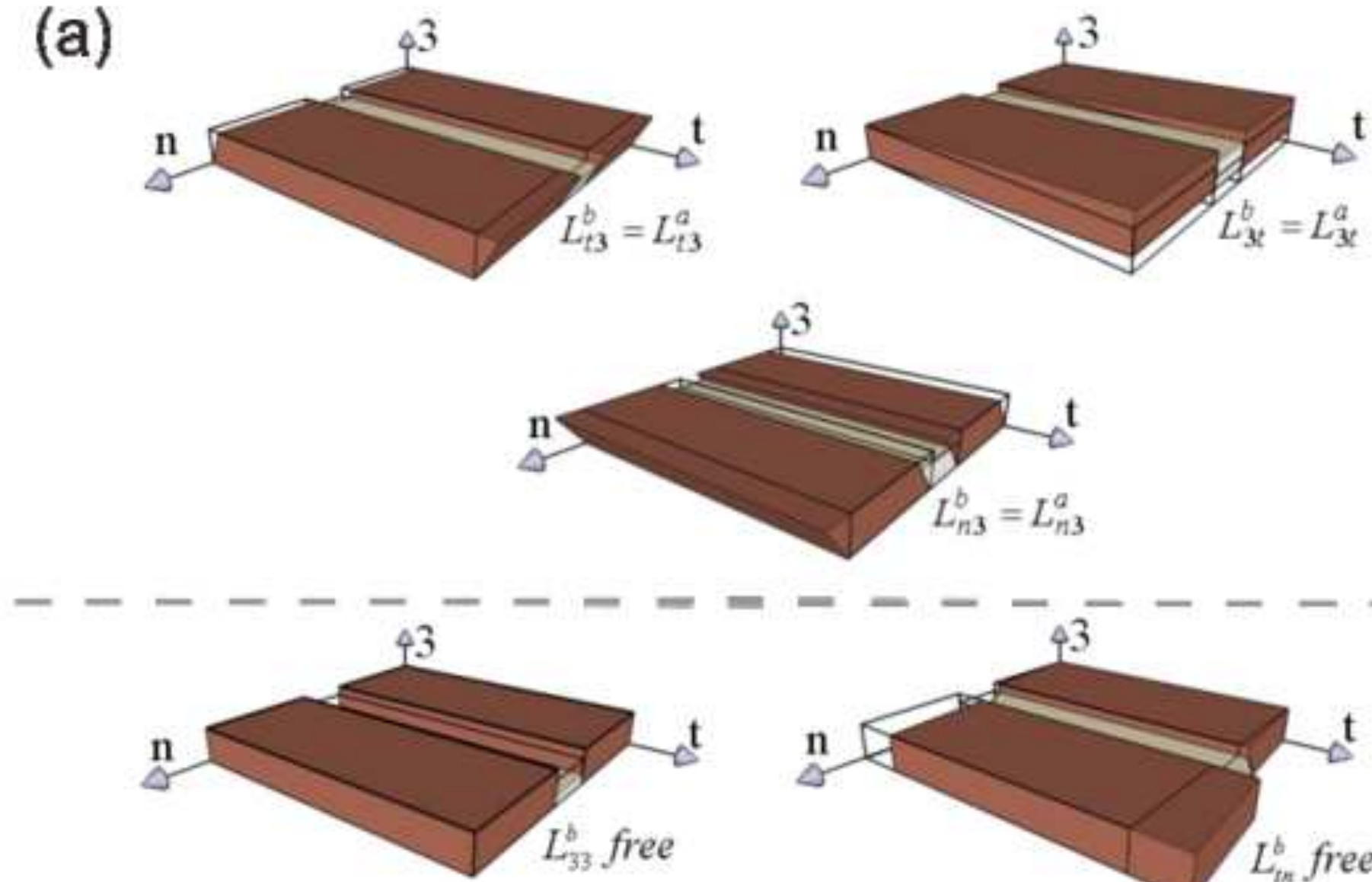

(b)
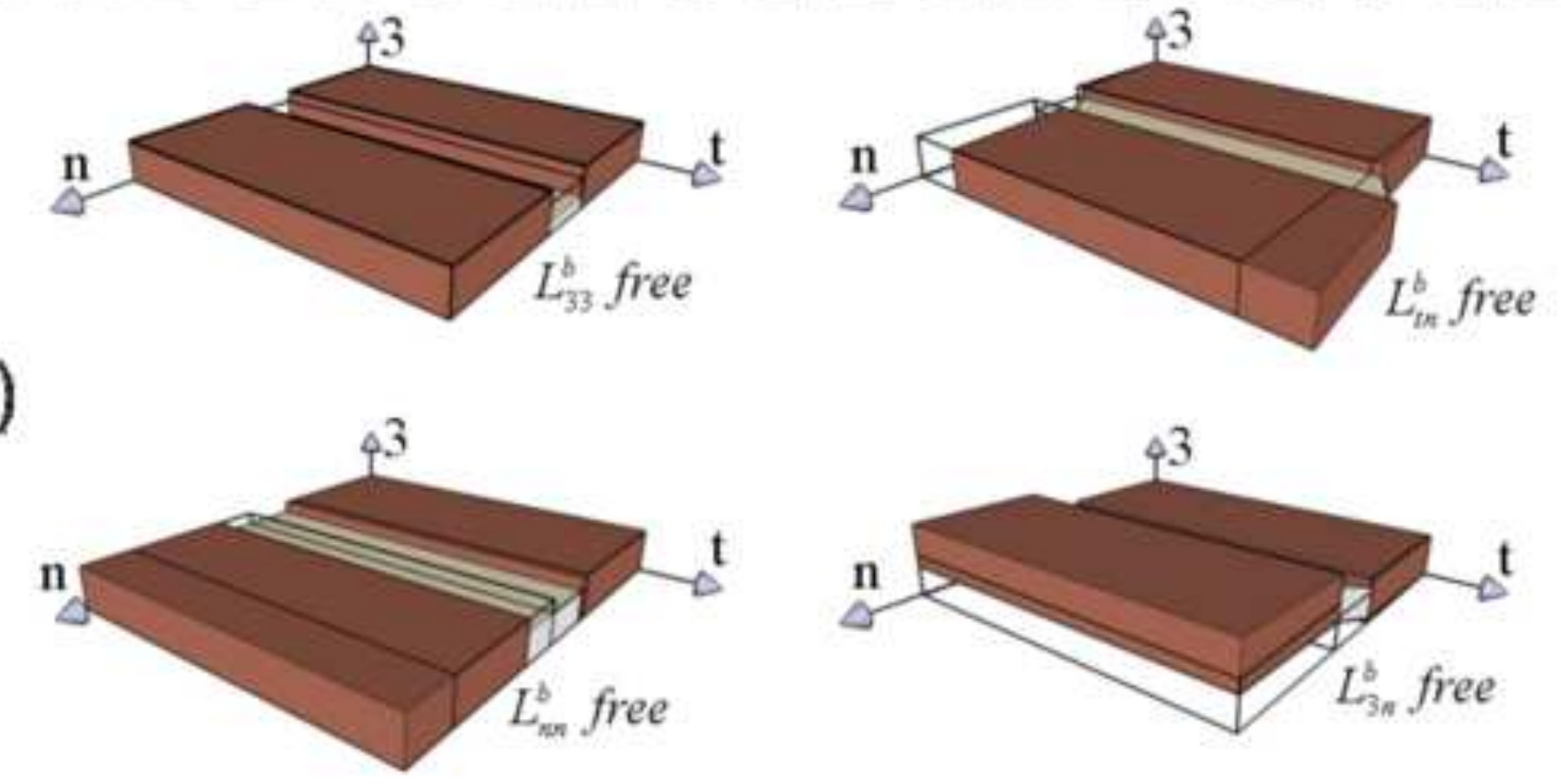

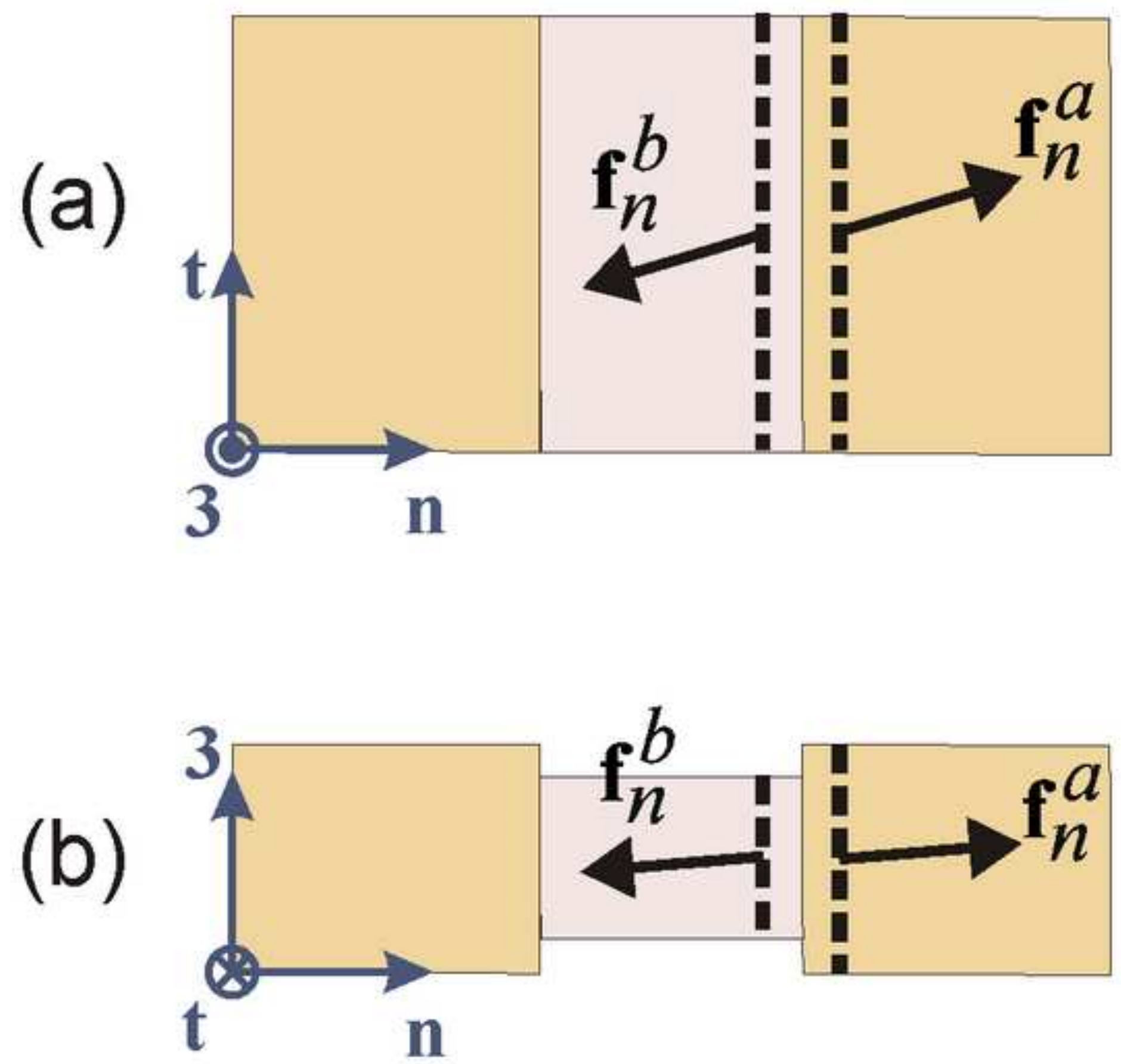

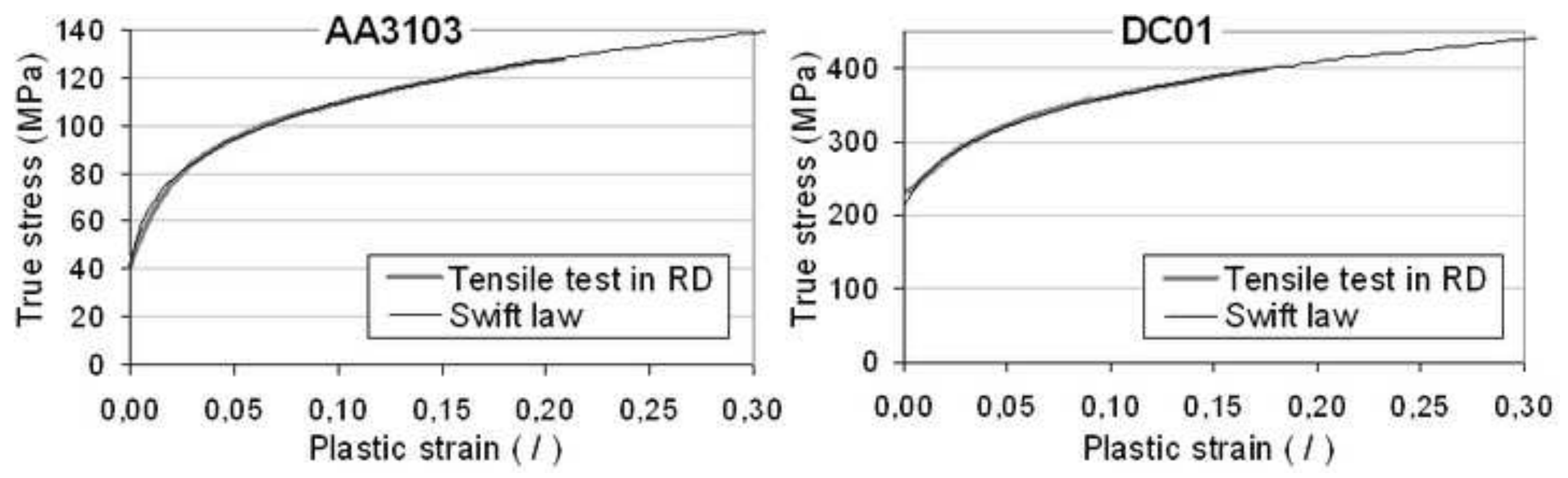


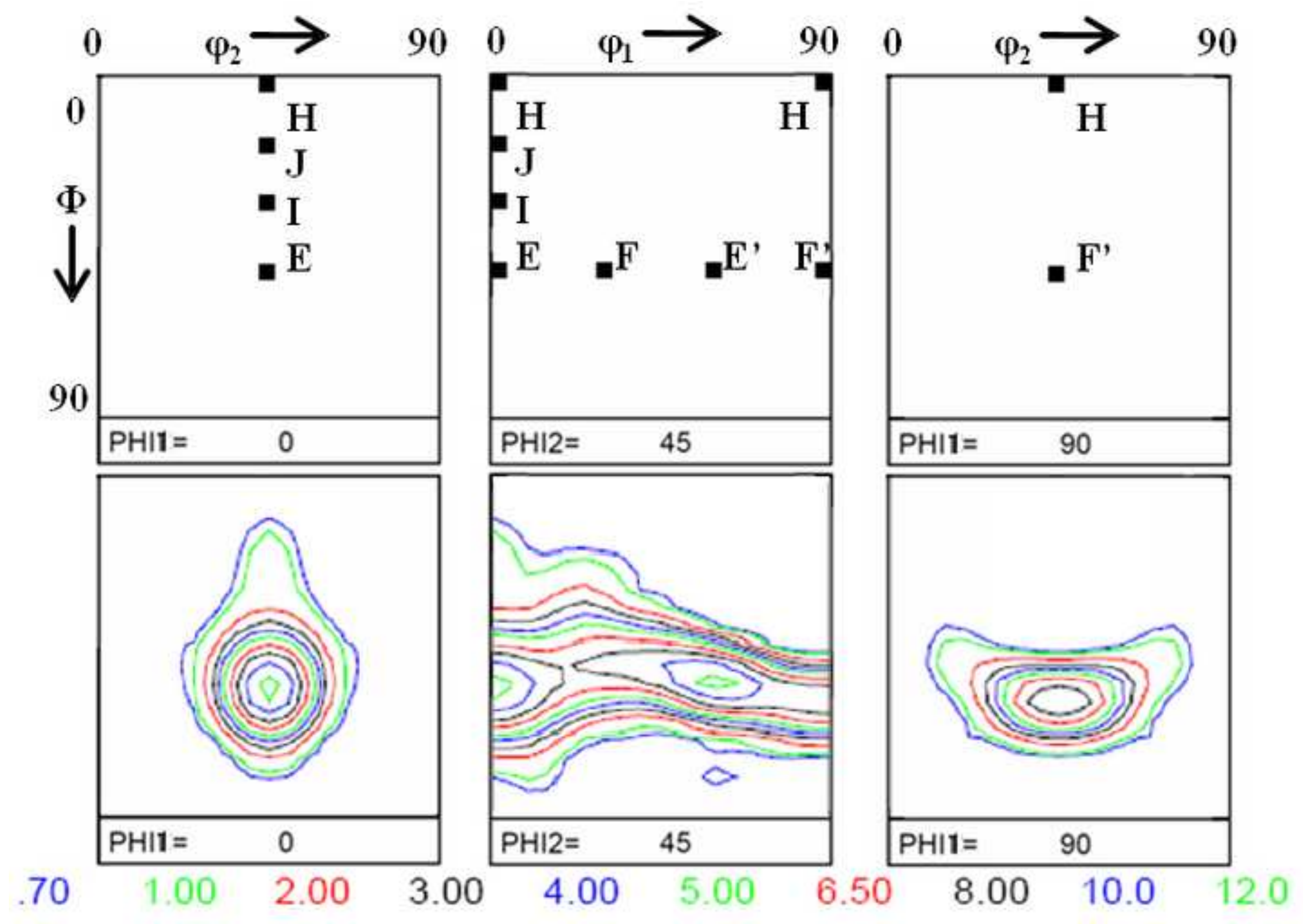


- Taylor

$\triangle$ 8th order Facet
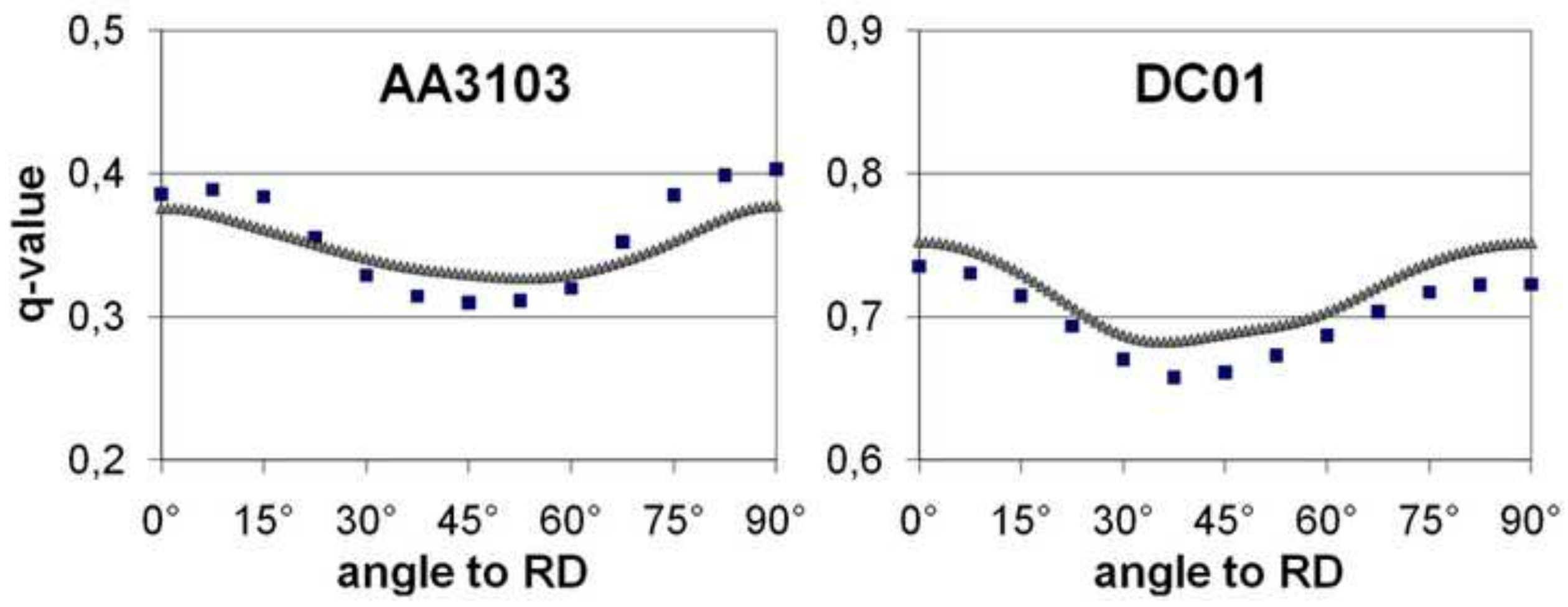


\section{-von Mises}

\#-Taylor (geom. method)

\section{-8th order Facet}
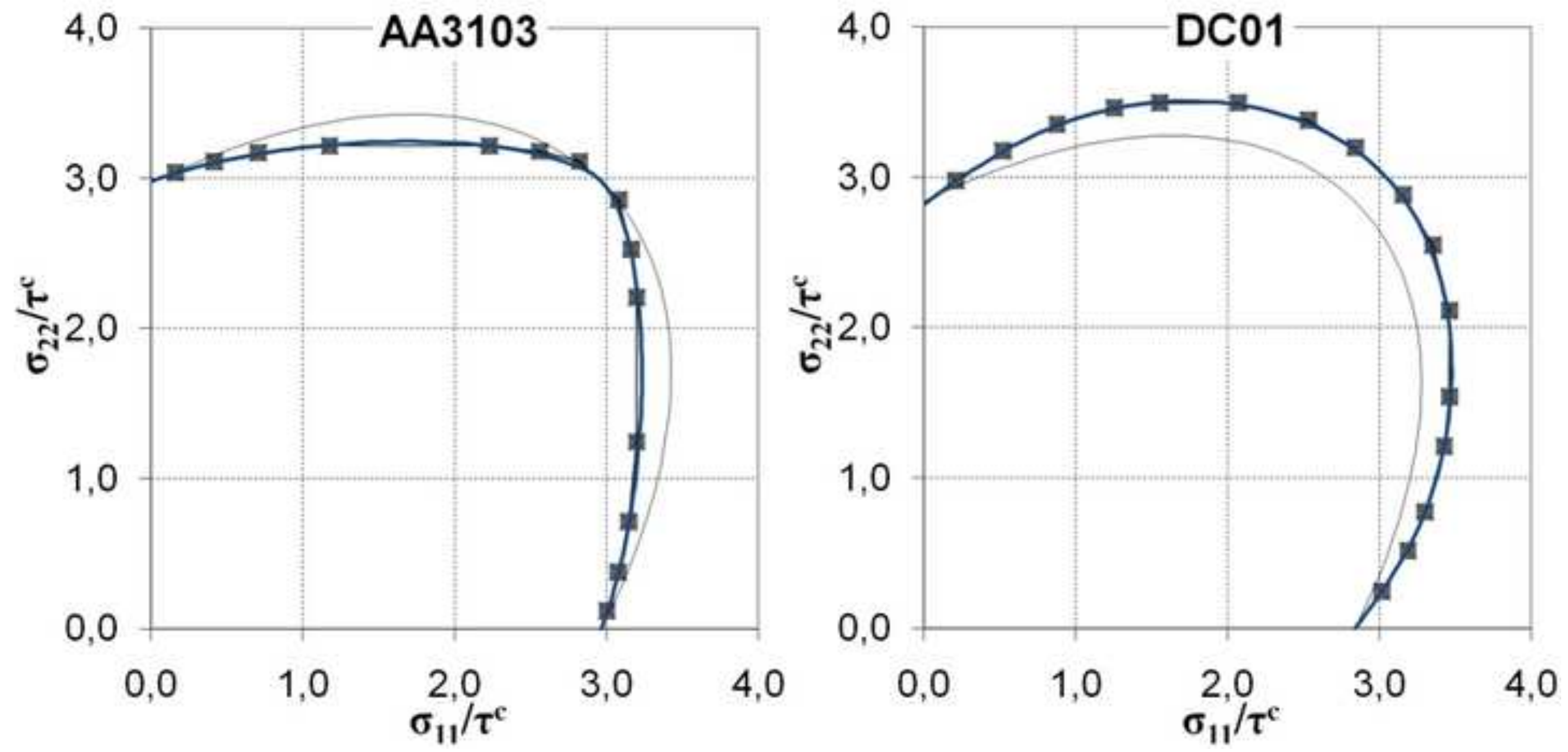
Figure 10

Click here to download high resolution image

\begin{tabular}{c|ccc}
\hline ij & Mises & Taylor (geom. method) & $8^{\text {th }}$ order Facet \\
\hline 23 & - & - & $\diamond$ \\
13 & - & $\square$ & $\square$ \\
12 & - & $\square$ & $\Delta$ \\
\hline
\end{tabular}
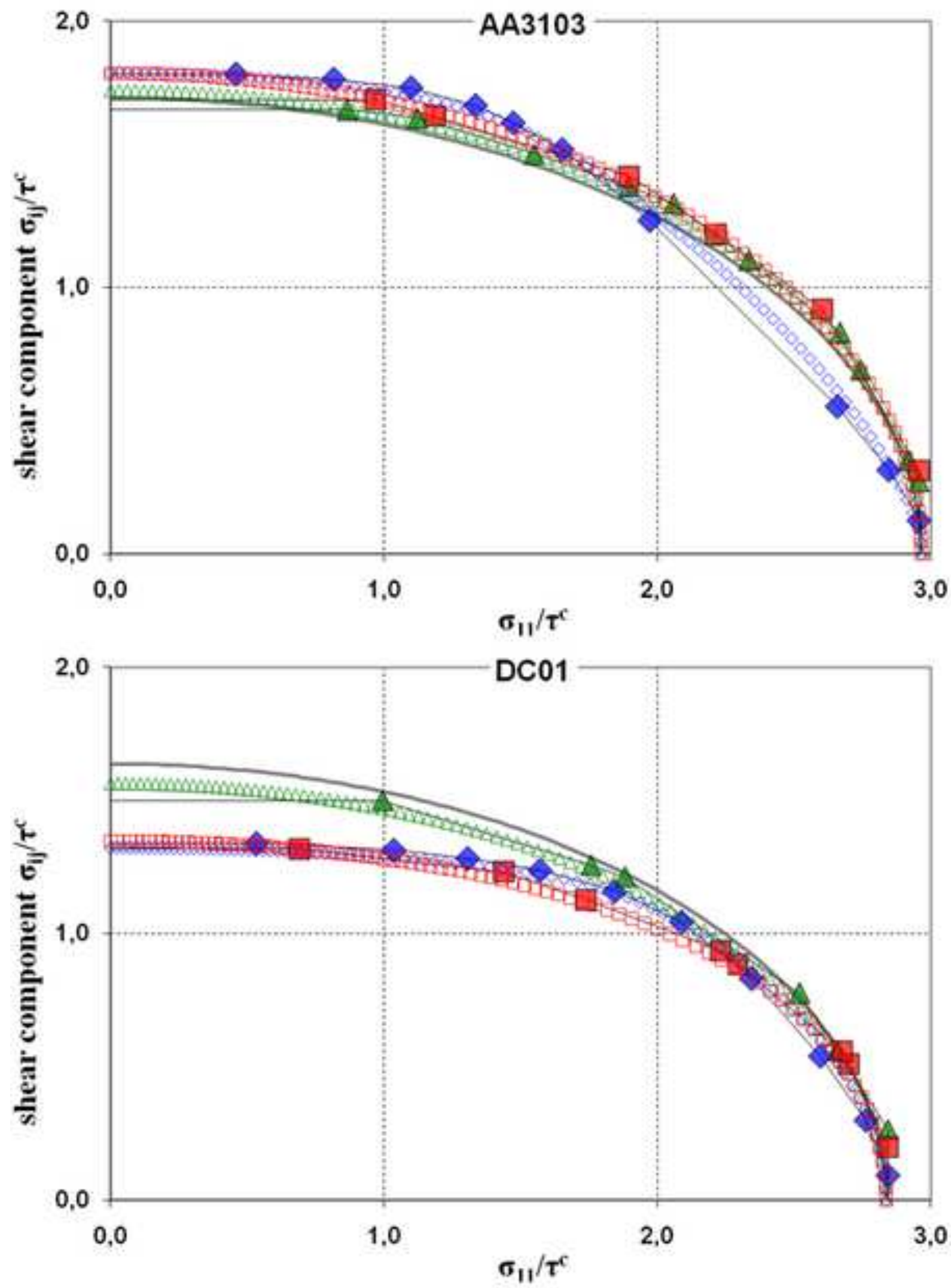


\begin{tabular}{c|cc}
\hline$\alpha_{R D}$ & Mises & $\boldsymbol{8}^{\text {th }}$ order Facet \\
\hline $\mathbf{0}^{\circ}$ & - & - \\
$\mathbf{4 5}^{\circ}$ & -- & - \\
$\mathbf{9 0}^{\circ}$ & $-\Theta-$ & - \\
\hline
\end{tabular}

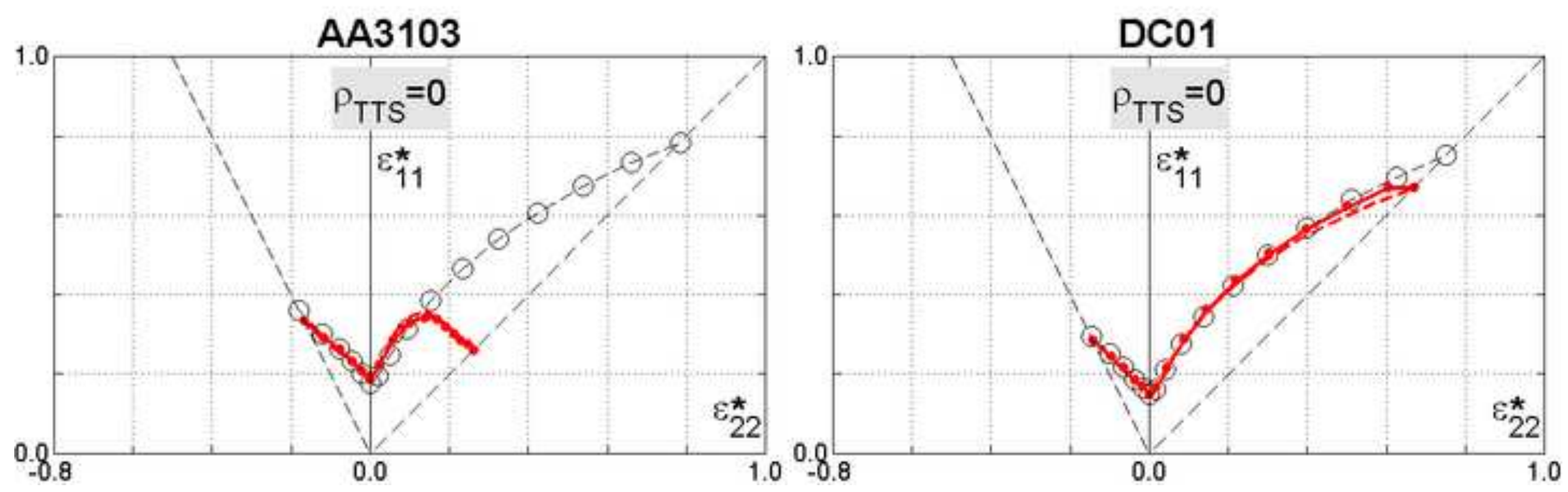




\begin{tabular}{c|cc}
\hline$\rho_{T T S}$ & Mises & $\boldsymbol{8}^{\text {th }}$ order Facet \\
\hline $\mathbf{0}$ & $-\vartheta-$ & - \\
$\mathbf{0 . 2}$ & $-\triangle-$ & - \\
$\mathbf{0 . 4}$ & $-\boxminus-$ & - \\
\hline
\end{tabular}

\section{AA3103}

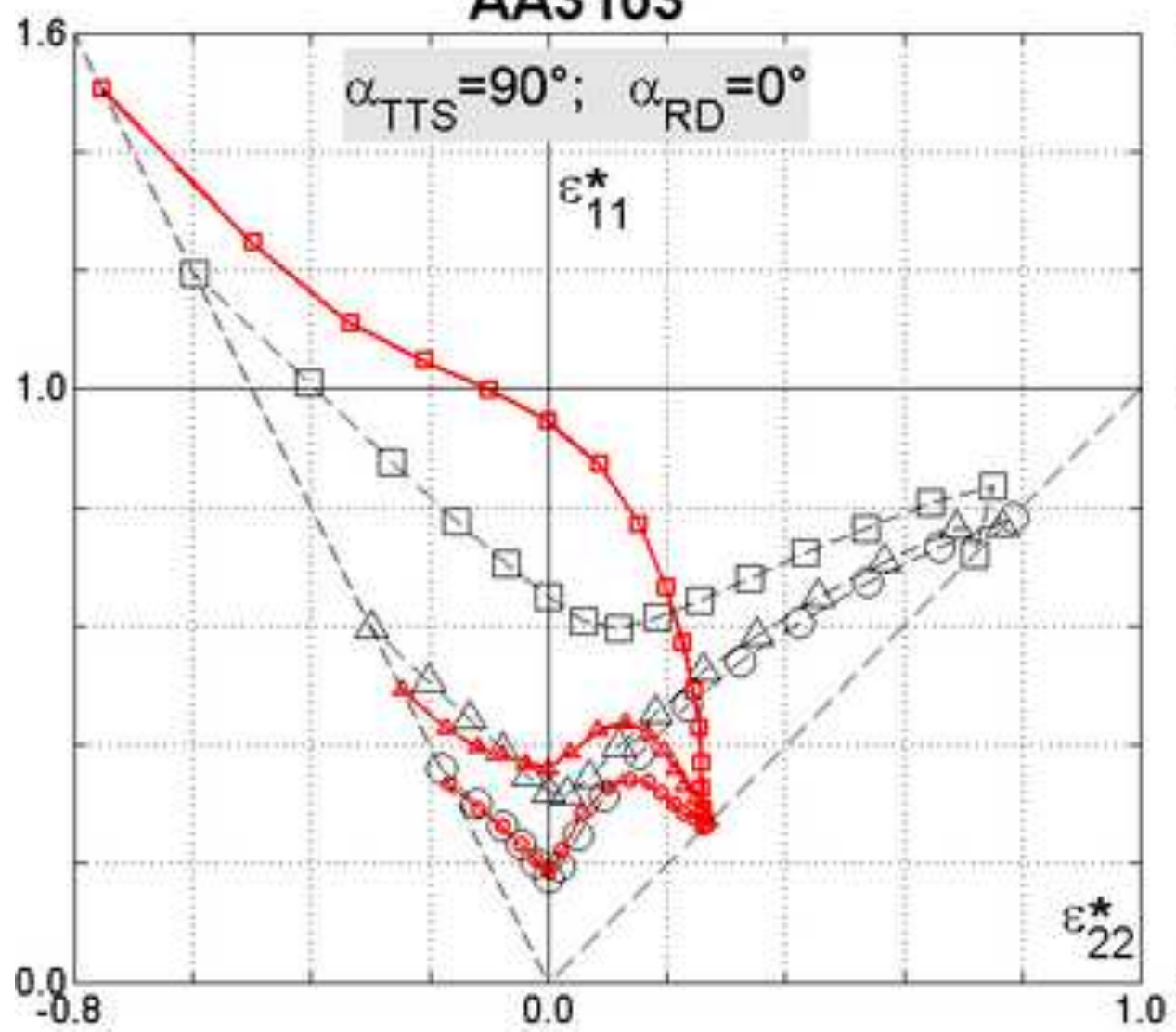

DC01

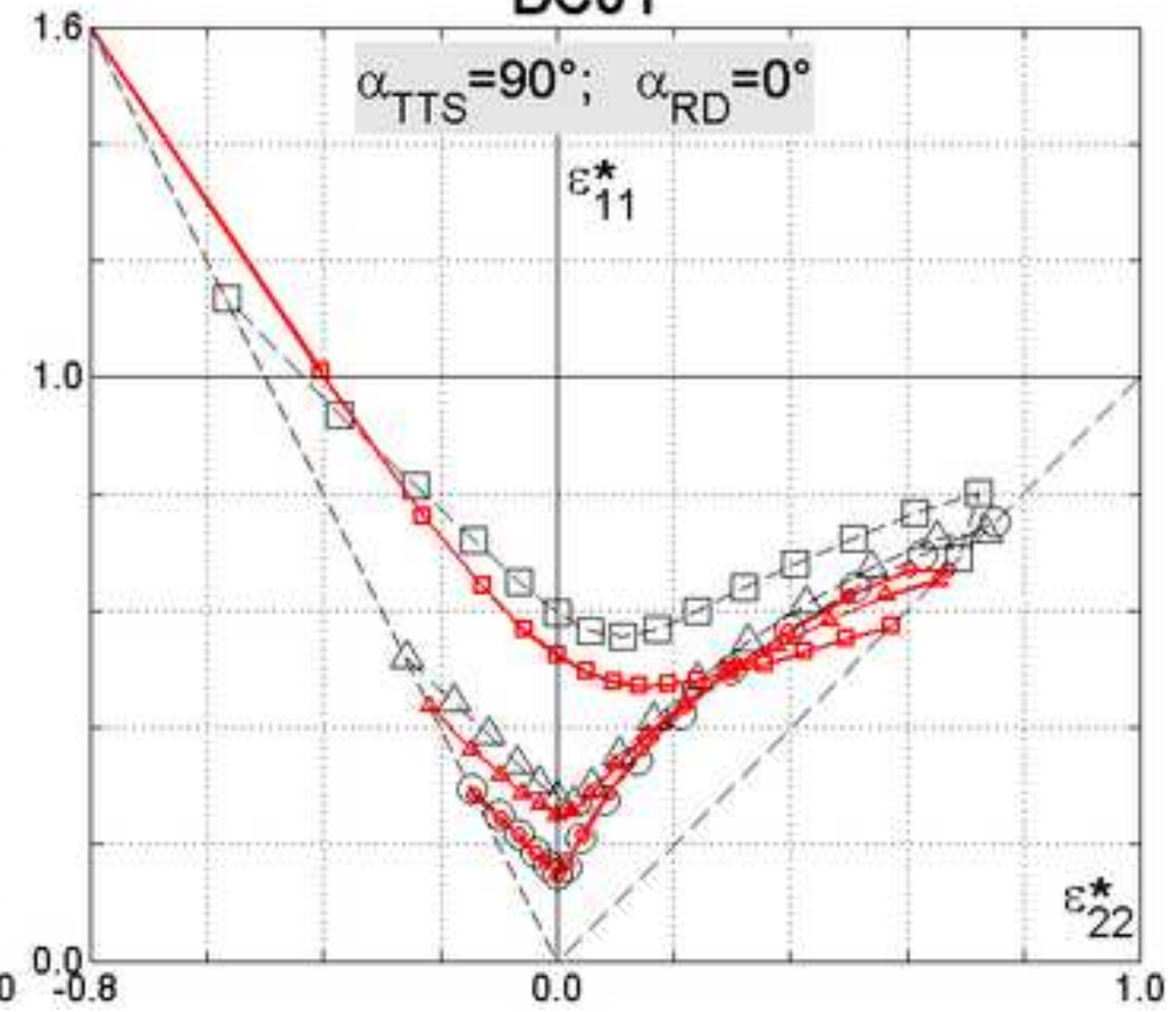




\begin{tabular}{c|cc}
\hline$\alpha_{R D}$ & Mises & $8^{\text {th }}$ order Facet \\
\hline $\mathbf{0}^{\circ}$ & $-\exists-$ & $\bullet$ \\
$\mathbf{4 5}$ & $-\exists-$ & -- \\
$\mathbf{9 0}^{\circ}$ & $-\exists-$ & - \\
\hline
\end{tabular}

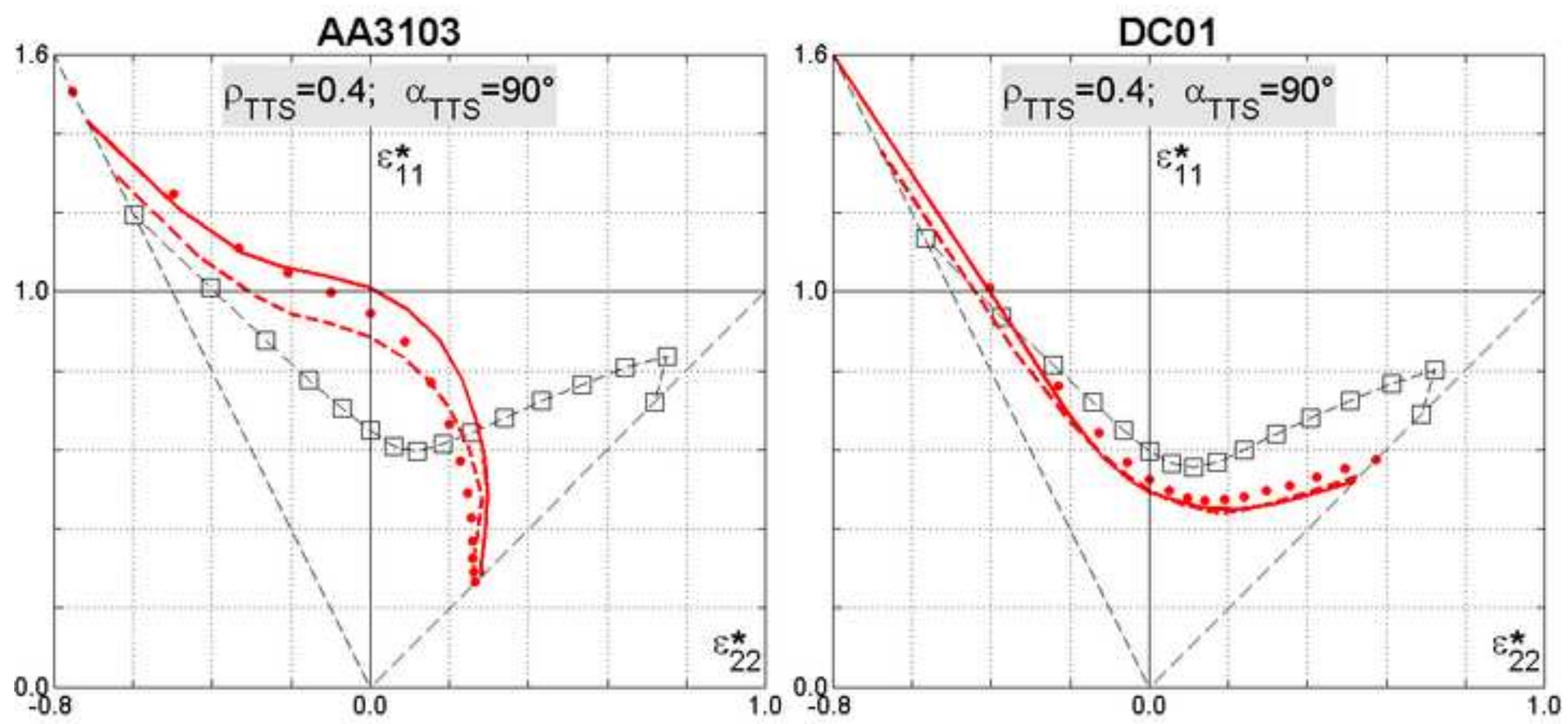




\begin{tabular}{c|c|c|c}
\hline$\alpha_{\text {TTS }}$ & $8^{\text {th }}$ order Facet & $\alpha_{\text {TTS }}$ & $8^{\text {th }}$ order Facet \\
\hline $0^{\circ}$ & - & $60^{\circ}$ & - \\
$15^{\circ}$ & - & $75^{\circ}$ & - \\
$30^{\circ}$ & - & $90^{\circ}$ & - \\
$45^{\circ}$ & - & & \\
\hline
\end{tabular}
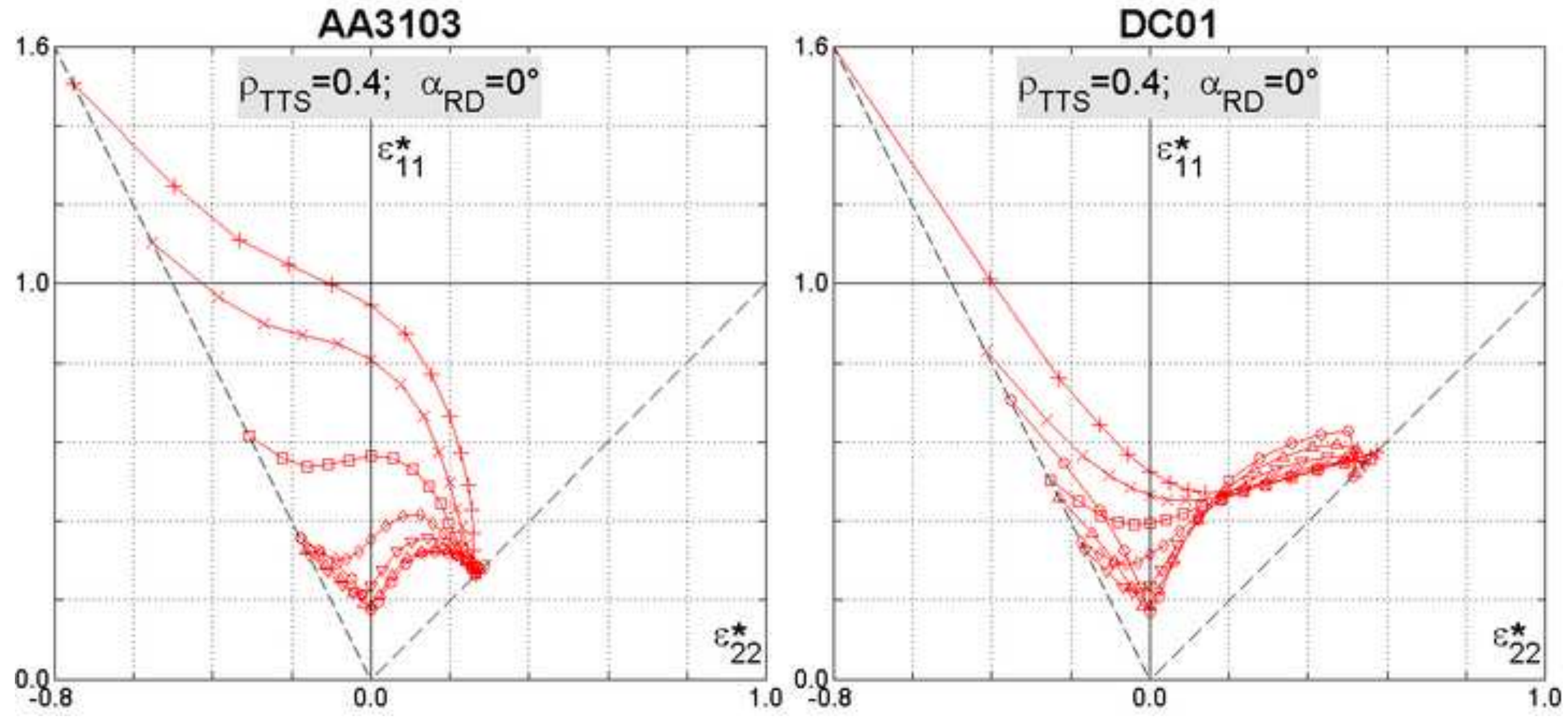
Table 1

Table 1

\begin{tabular}{|c|c|c|}
\cline { 2 - 3 } \multicolumn{1}{c|}{} & AA3103 & DC01 \\
\hline$K[\mathrm{MPa}]$ & 180.0 & 547.0 \\
\hline$\varepsilon_{0}$ & 0.00068 & 0.00544 \\
\hline$n$ & 0.218 & 0.184 \\
\hline
\end{tabular}

